SCIREA Journal of Civil Engineering and

Building Construction

http://www.scirea.org/journal/CEBC

October 24, 2021

SCIREA

Volume 6, Issue 1, February 2021

\title{
FOUNDATION FOR PRIMARY AIR FAN OF SPEED 1490 RPM - STUDY OF DYNAMIC BEHAVIOR
}

\section{Nulu Reddeppa ${ }^{1 *}$, B Jayarami Reddy ${ }^{2}$, H Sudarsana Rao ${ }^{3}$}

${ }^{1}$ Ph.D. Scholar, Dept. of Civil Engineering, JNTUA, Anantapuramu, Andhra Pradesh, India

${ }^{2}$ Head of the department, Dept. of Civil Engineering, YSR Engineering. College of YVU, Proddatur, Andhra Pradesh, India

${ }^{3}$ Professor, Dept. of Civil Engineering, JNTUA, Anantapuramu, Andhra Pradesh, India

*Corresponding Author- Nulu Reddeppa, email: nreddeppa@gmail.com

ORCID of Nulu Reddeppa: 0000-0002-6828-6960

\begin{abstract}
In general Primary Air fan (PA Fan) foundation consist of a block foundation / semi framed foundation with heavy concrete mass. Its dynamic behavior is studied using Barkan's method by most of the engineering practitioners. This method has limitations in providing wholistic details of foundation behavior like transient stage behavior, response at specific part of the foundation etc. However, in this study a three-dimensional Finite Element model is used to study its dynamic behavior. ANSYS software is used for modeling the foundation with three-dimensional Finite Elements. Appropriate soil structure interaction is resembled with suitable elements available in ANSYS element library. Natural frequencies of the foundation system and displacement amplitudes are obtained using the above model. Mode shapes of the various modes are plotted. Foundation response for equipment startup and shut down conditions are studied by plotting displacement amplitudes w.r.t frequency variation.
\end{abstract}

Keywords: PA Fan Foundation, Harmonic Analysis, Barkan's Method, ANSYS, Frequency 


\section{INTRODUCTION}

PA fan foundation is one of the critical foundation in thermal power plants. Primary Air fans of this capacity are used in thermal power plants of size $800 \mathrm{MW}$. Abnormal fan vibrations may lead to operational problems, shutdowns, and curtailed operations of power plants. The safe and optimum design of foundation for this critical equipment is an important aspect in power plants. Therefore, the analysis of large structural concrete foundations for fans presents a challenge to a wide variety of industrial plants [1]. Concrete foundation cracks result not only from mechanical defects which cannot be completely resolved by plant personnel (e.g. imbalance and misalignment), but also from type of loading, speed of rotors, and cyclic and dynamic loading. Fan vibration, caused by the mentioned reasons as well as the frequency resonance of the dynamic load, can reduce the safety factor of fan foundation [2,3]. Simply checking the validity of the foundation design for the stationary situation (as it often happens in practice) might not be enough to produce a proper foundation design [4].

Evolution of software's based on Finite element method's such as Staad.Pro, SAP, ANSYS, Nastran etc.., paved the way to understand the behaviour of these block foundations under dynamic loads under different operating conditions. From output results of these software's one can read the displacements / velocity amplitudes at different points on the foundation. This is helping practitioners to predict the behaviour of foundation more precisely during equipment operating conditions. The finite element model (FEM) is a beneficial method to include all parameters without the construction of a full-scale foundation [5]. Research has shown that the first and second natural frequencies obtained from a stiffness matrix with coupled lateral behavior provided very good correspondence with the FEM of the foundation, especially when the effects of inertia on the foundation were negligible [6]

\section{GEOMETRY AND BASIC DATA}

The geometry is considered as per machine manufacturer's foundation input drawings. It can be seen from the geometry shown in figure 2.0, that the fan foundation is a block foundation supported by framed foundation.

\subsection{Material properties}

Table-1 Material properties of concrete

\begin{tabular}{|c|c|c|}
\hline Material & Property & Value \\
\hline \multirow{4}{*}{$\begin{array}{l}\text { Concrete, } \\
\text { M35 }\end{array}$} & Density, KN/Cum & 25 \\
\hline & $\begin{array}{l}\text { Characteristic Strength, } \\
\mathrm{N} / \mathrm{mm}^{2}\end{array}$ & 35 \\
\hline & $\begin{array}{ll}\text { Upper } & \text { Modulus } \\
\text { Elasticity, } \mathrm{N} / \mathrm{mm}^{2} & \end{array}$ & $\begin{array}{l}40000 \\
\text { (Dynamic) }\end{array}$ \\
\hline & $\begin{array}{l}\text { Lower Modulus of } \\
\text { Elasticity, } \mathrm{N} / \mathrm{mm} 2\end{array}$ & $\begin{array}{l}33722 \\
\text { (Dynamic) }\end{array}$ \\
\hline
\end{tabular}

Dynamic young's modulus of concrete will have a range also actual concrete grade that will used at construction may vary on upper side. Hence the FEM analysis is required to be done for the lower and upper possible values of Young's modulus of concrete. M30 grade of concrete is used for pile.

\subsection{MACHINE DATA}

The machine and foundation arrangement with the loads are specified in machine manufacturer's drawings / documents. The salient features of the machine are summarized as below.

Total Fan Weight $=373.3 \mathrm{kN}$
Motor weight $=155 \mathrm{kN}$
Weight of rotating parts in fan $=29.40$
$\mathrm{kN}$
Weight of rotating parts in motor= $\mathrm{kN}$

Self-weight of the Foundation $\cong 3091$ $\mathrm{kN}$

weight ratio of Foundation to Machine $=5.85>2.5$

weight ratio of Foundation to rotor $=44.538>2.5$

\subsection{SOFTWARE REFERENCE}

ANSYS/Structural 13.0 ver, ANSYS, Inc., USA is renowned and is in practice in the industry both nationally and internationally for similar type of structural dynamic analysis for PA Fan foundations. Consistent units of Newton, Meter are used in the present analysis.

\section{FEM MODELING OF STRUCTURE}

Solid brick finite elements are used to represent the geometry of fan foundation for dynamic analysis. The solid model is built in ANSYS software based on this geometry and then the finite element is created by meshing using solid elements "solid186" of ANSYS element library. The volume mesh contains brick element shapes of the solid186 shown below. Solid186 element has three degrees of freedom in three linear directions. The foundation is provided with piles spring supports estimated as per the soil characteristics of the site. 

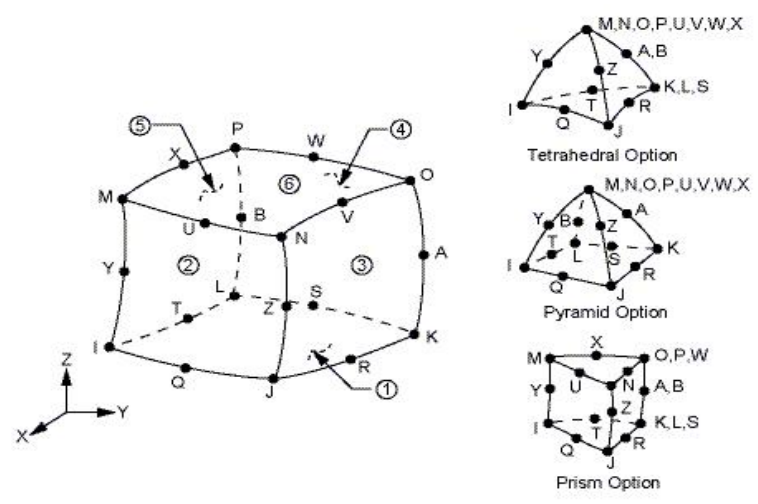

Figure.1 Solid186 Element Geometry

The following sections describe the analysis/design methodology including loading consideration and finally summarize the results.

\subsection{MODAL ANALYSIS - NATURAL FREQUENCIES}

The Mode-Frequency analysis for natural frequency and mode shape determination is carried out in ANSYS. The assumptions made in this analysis are:

a) The structure has no time varying forces, displacements, pressures, or temperatures applied, which means that this is free vibration analysis.

b) There is no damping in the structural system.

c) The structure has constant stiffness and mass effects.

3D MASS 21 element (from ANSYS element library) is used to represent machine mass application points on foundation block. COMBIN14 is used to model vertical and lateral pile springs on the foundation.

The natural frequencies are requested for first 50 modes of vibration. But only 10 modes are present in the foundation system below $50 \mathrm{~Hz}$ and they are tabulated below. Refer to ANSYS output in Section 5 for natural frequency output and mode participation factor table.

\subsection{Dynamic stiffness Calculations}

Data:

Length of the pile $\mathrm{L}$

Diameter of the pile D

$=$

$=$

$49 \mathrm{~m}$

Radius of the pile $\mathrm{R}$

$=$

$0.75 \mathrm{~m}$

$0.375 \mathrm{~m}$

Pile Slenderness $=\mathrm{L} / \mathrm{R}$ 130.7

Weighted average Young's modulus of the soil along the pile length to be calculated as per site condition. However, the same is considered as $206.8104 \mathrm{MPa}$ in the present analysis.
Ep

$$
=27386 \mathrm{MPa}=5000 * \sqrt{f c k}
$$

$\begin{array}{lll}\text { Poisson's Ratio } \mu & = & 0.281 \\ \text { Unit weight of the concrete } & = & 25 \mathrm{kN} / \mathrm{m}^{3}\end{array}$

Shear Modulus,

$$
\text { Gs }=\frac{E s}{2(1+\mu)} \quad=\quad 94.288 \mathrm{MPa}
$$

Unit weight of the soil, $\gamma \mathrm{s}=$

$18 \mathrm{kN} / \mathrm{m}^{3}$

Shear wave velocity,

$V s=\sqrt{\frac{G s g}{r_{s}}}$

$=7.1685 \mathrm{~m} / \mathrm{sec}$

[3]

Compression wave velocity

$V c=\sqrt{\frac{E p g}{r_{p}}}$

$=103.66 \mathrm{~m} / \mathrm{sec}$

[4]

$\mathrm{Vs} / \mathrm{Vc}=0.069$

Stiffness Factor $\mathrm{f}_{18,1} \quad=0.036$

Radius of the pile $\quad=375 \mathrm{~mm}$

Vertical Stiffness is governed by the soil at the bottom of the pile which is dense sand.

$$
\begin{aligned}
\mathrm{Kz} & =\left(\frac{E p \cdot A}{Y_{o}}\right) \cdot f 18,1 \\
& =1161954283 \mathrm{~N} / \mathrm{m}
\end{aligned}
$$

Since the Lateral Stiffness is governed by the top pf the pile which is dense sand.

$$
\begin{array}{ll}
\text { Young's Modulus Es } & =123.52 \mathrm{MPa} \\
\text { Poisson's Ratio } \mu & =0.258
\end{array}
$$

Shear Modulus,

$$
\begin{aligned}
G S & =\frac{E s}{2(1+\mu)} \\
& =49.094 \mathrm{MPa}
\end{aligned}
$$

Shear wave velocity,

$$
\begin{aligned}
\mathrm{Vs} & =\sqrt{G s g / \gamma s} \\
& =5.17 \mathrm{~m} / \mathrm{sec}
\end{aligned}
$$

Compression wave velocity

$$
\begin{aligned}
& V c=\sqrt{\frac{E p g}{\gamma_{p}}}=103.66 \mathrm{~m} / \mathrm{sec} \\
& \mathrm{Vs} / \mathrm{Vc}=5.173 / 103.7 \quad=0.0498
\end{aligned}
$$

From table 5-2 of the Design of structures and foundation for vibrating machines by Suresh Arya, Michel O' Neil, Gorge Pincus. 


$$
\mathrm{f}_{11,1}=0.03591
$$

Moment of Inertia (I) $=15 \mathrm{E}+09$

$$
\begin{aligned}
\text { Horizontal Stiffness Kx } & =\left(\frac{E p \cdot A}{Y_{o}}\right) \cdot f 11,1 \\
& =289757370.5 \mathrm{~N} / \mathrm{m}
\end{aligned}
$$

Modal Analysis is performed for both the static pile stiffness and dynamic pile stiffness. The frequency separation criteria is met for both the cases.

\section{STRUCTURAL MODEL SKETCHES}

Below sketches shows the FEM model of ID fan foundation system generated using the ANSYS software.

\subsection{ANSYS model sketches}

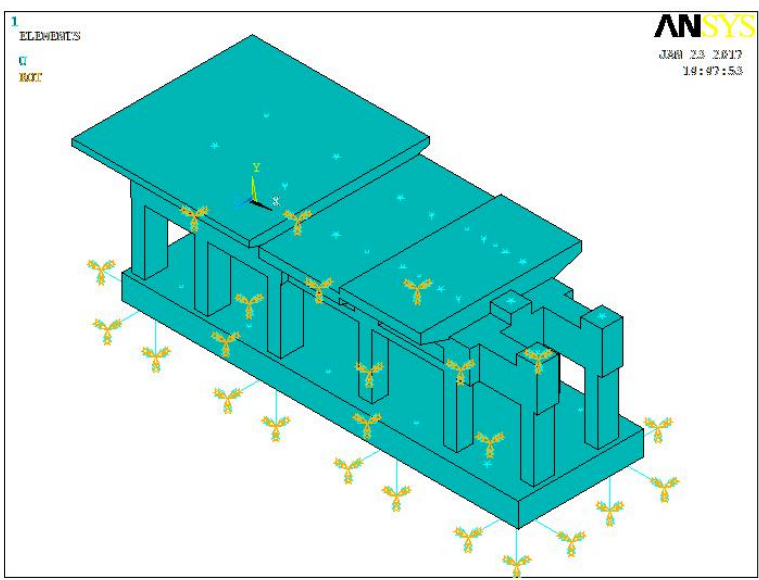

Figure 2. ANSYS - solid model

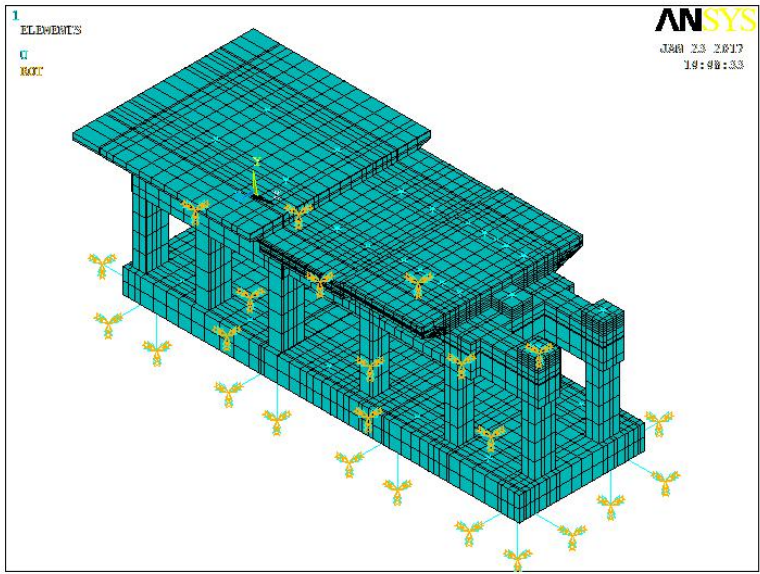

Figure 3. ANSYS - solid model - element mesh

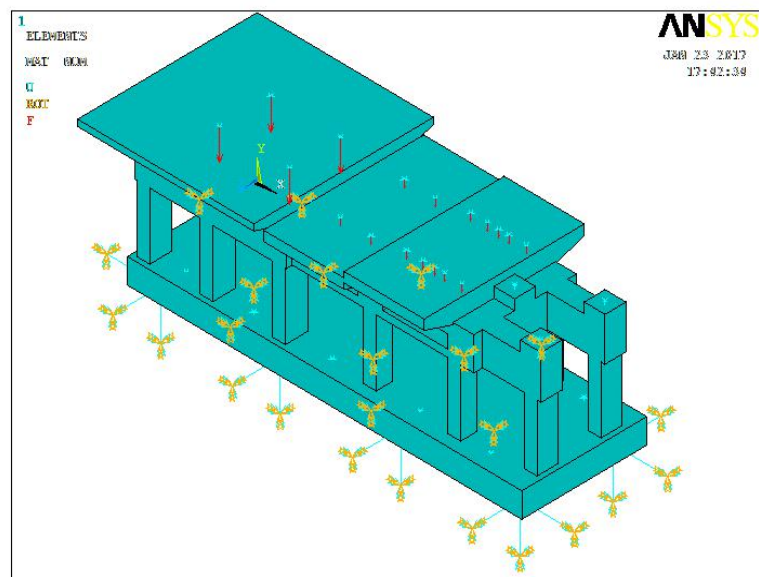

Figure 4. ANSYS - solid model - applied boundary conditions

\section{ANALYSIS OUTPUT}

\subsection{ANSYS - natural frequencies for upper "E"}

Table 2. Participation factor calculation $\mathrm{X}$ direction

\begin{tabular}{cccccccc}
\hline MODE & $\begin{array}{c}\text { FREQUENC } \\
\mathbf{Y}\end{array}$ & PERIOD & $\begin{array}{c}\text { PARTIC. } \\
\text { FACTOR }\end{array}$ & RATIO & $\begin{array}{c}\text { EFFECTIV } \\
\text { E MASS }\end{array}$ & $\begin{array}{c}\text { CUMULATI } \\
\text { VE MASS } \\
\text { FRACTION }\end{array}$ & $\begin{array}{c}\text { RATIO } \\
\text { EFF.MASS } \\
\text { TO TOTAL } \\
\text { MASS }\end{array}$ \\
\hline 1 & 6.61 & 0.151 & $-1.09 \mathrm{E}-05$ & 0.000 & $1.19 \mathrm{E}-10$ & $2.72 \mathrm{E}-16$ & $2.72 \mathrm{E}-16$ \\
\hline 2 & 8.68 & 0.115 & $6.20 \mathrm{E}+02$ & 1.000 & 384170 & $8.79 \mathrm{E}-01$ & 0.878621 \\
\hline 3 & 11.27 & 0.089 & $8.07 \mathrm{E}-06$ & 0.000 & $6.51 \mathrm{E}-11$ & $8.79 \mathrm{E}-01$ & $1.49 \mathrm{E}-16$ \\
\hline 4 & 18.32 & 0.055 & $-1.19 \mathrm{E}+01$ & 0.019 & 142.398 & $8.79 \mathrm{E}-01$ & $3.26 \mathrm{E}-04$ \\
\hline 5 & 18.97 & 0.053 & $-9.25 \mathrm{E}-06$ & 0.000 & $8.55 \mathrm{E}-11$ & $8.79 \mathrm{E}-01$ & $1.96 \mathrm{E}-16$ \\
\hline 6 & 20.51 & 0.049 & $2.25 \mathrm{E}+02$ & 0.362 & $5.05 \mathrm{E}+04$ & $9.94 \mathrm{E}-01$ & $1.15 \mathrm{E}-01$ \\
\hline 7 & 26.22 & 0.038 & $4.92 \mathrm{E}+01$ & 0.079 & $2.42 \mathrm{E}+03$ & $1.00 \mathrm{E}+00$ & $5.55 \mathrm{E}-03$ \\
\hline 8 & 26.91 & 0.037 & $4.57 \mathrm{E}-05$ & 0.000 & $2.09 \mathrm{E}-09$ & $1.00 \mathrm{E}+00$ & $4.78 \mathrm{E}-15$ \\
\hline 9 & 42.34 & 0.024 & $3.31 \mathrm{E}+00$ & 0.005 & $1.10 \mathrm{E}+01$ & $1.00 \mathrm{E}+00$ & $2.51 \mathrm{E}-05$ \\
\hline 10 & 43.61 & 0.023 & $6.57 \mathrm{E}-05$ & 0.000 & $4.32 \mathrm{E}-09$ & $1.00 \mathrm{E}+00$ & $9.87 \mathrm{E}-15$ \\
\hline sum & & & & & $4.37 \mathrm{E}+05$ & & $1.00 \mathrm{E}+00$ \\
\hline
\end{tabular}

Table 3. Participation factor calculation Y direction

\begin{tabular}{cccccccc}
\hline MODE FREQUENCY PERIOD & $\begin{array}{c}\text { PARTIC. } \\
\text { FACTOR }\end{array}$ & RATIO & $\begin{array}{c}\text { EFFECTIV } \\
\text { E MASS }\end{array}$ & $\begin{array}{c}\text { CUMULATI } \\
\text { VE MASS } \\
\text { FRACTION }\end{array}$ & $\begin{array}{c}\text { RATIO } \\
\text { EFF.MASS } \\
\text { TO TOTAL } \\
\text { MASS }\end{array}$ \\
\hline 1 & & & & & & & \\
\hline 2 & 8.61 & 0.151 & $1.11 \mathrm{E}-05$ & 0.000 & $1.24 \mathrm{E}-10$ & $2.83 \mathrm{E}-16$ & $2.83 \mathrm{E}-16$ \\
\hline 3 & 11.27 & 0.115 & $3.08 \mathrm{E}+00$ & 0.005 & 9.51343 & $2.18 \mathrm{E}-05$ & $2.1758 \mathrm{E}-05$ \\
\hline 4 & 18.32 & 0.059 & $-4.18 \mathrm{E}-06$ & 0.000 & $1.75 \mathrm{E}-11$ & $2.18 \mathrm{E}-05$ & $3.99 \mathrm{E}-17$ \\
\hline 5 & 18.97 & $0.54 \mathrm{E}+02$ & 1.000 & 427678 & $9.80 \mathrm{E}-01$ & $9.78 \mathrm{E}-01$ \\
\hline 6 & 20.51 & 0.049 & $1.14 \mathrm{E}-04$ & 0.000 & $1.30 \mathrm{E}-08$ & $9.80 \mathrm{E}-01$ & $2.98 \mathrm{E}-14$ \\
\hline 7 & 26.22 & 0.038 & $-2.99 \mathrm{E}+01$ & 0.048 & $9.89 \mathrm{E}+02$ & $9.82 \mathrm{E}-01$ & $2.26 \mathrm{E}-03$ \\
\hline 8 & 26.91 & 0.037 & $7.63 \mathrm{E}-06$ & 0.000 & $5.97 \mathrm{E}+02$ & $9.84 \mathrm{E}-01$ & $2.05 \mathrm{E}-03$ \\
\hline 9 & 42.34 & 0.024 & $8.34 \mathrm{E}+01$ & 0.128 & $6.96 \mathrm{E}+03$ & $1.00 \mathrm{E}-01$ & $1.33 \mathrm{E}-16$ \\
\hline 10 & 43.61 & 0.023 & $1.54 \mathrm{E}-03$ & 0.000 & $2.39 \mathrm{E}-06$ & $1.00 \mathrm{E}+00$ & $1.59 \mathrm{E}-02$ \\
\hline sum & & & & & $4.37 \mathrm{E}+05$ & & $9.98 \mathrm{E}-01$ \\
\hline
\end{tabular}

Table 4. Participation factor calculation $\mathrm{z}$ direction 


\begin{tabular}{cccccccc}
\hline MODE FREQUENCY PERIOD & $\begin{array}{c}\text { PARTIC. } \\
\text { FACTOR }\end{array}$ & RATIO & $\begin{array}{c}\text { EFFECTIV } \\
\text { E MASS }\end{array}$ & $\begin{array}{c}\text { CUMULATI } \\
\text { VE MASS } \\
\text { FRACTION }\end{array}$ & $\begin{array}{c}\text { RATIO } \\
\text { EFF.MASS } \\
\text { TO TOTAL } \\
\text { MASS }\end{array}$ \\
\hline 1 & 6.61 & 0.151 & $5.78 \mathrm{E}+02$ & 1.000 & $3.35 \mathrm{E}+05$ & $7.65 \mathrm{E}-01$ & $7.65 \mathrm{E}-01$ \\
\hline 2 & 8.68 & 0.115 & $1.14 \mathrm{E}-05$ & 0.000 & $1.295 \mathrm{E}-10$ & $7.65 \mathrm{E}-01$ & $2.9618 \mathrm{E}-16$ \\
\hline 3 & 11.27 & 0.089 & $3.98 \mathrm{E}+01$ & 0.069 & $1.58 \mathrm{E}+03$ & $7.69 \mathrm{E}-01$ & $3.62 \mathrm{E}-03$ \\
\hline 4 & 18.32 & 0.055 & $-6.51 \mathrm{E}-05$ & 0.000 & $4.241 \mathrm{E}-09$ & $7.69 \mathrm{E}-01$ & $9.70 \mathrm{E}-15$ \\
\hline 5 & 18.97 & 0.053 & $3.18 \mathrm{E}+02$ & 0.549 & $1.01 \mathrm{E}+05$ & $1.00 \mathrm{E}+00$ & $2.31 \mathrm{E}-01$ \\
\hline 6 & 20.51 & 0.049 & $4.09 \mathrm{E}-06$ & 0.000 & $1.67 \mathrm{E}-11$ & $1.00 \mathrm{E}+00$ & $3.83 \mathrm{E}-17$ \\
\hline 7 & 26.22 & 0.038 & $4.56 \mathrm{E}-06$ & 0.000 & $2.07 \mathrm{E}-11$ & $1.00 \mathrm{E}+00$ & $4.75 \mathrm{E}-17$ \\
\hline 8 & 26.91 & 0.037 & $-8.15 \mathrm{E}-01$ & 0.001 & $6.65 \mathrm{E}-01$ & $1.00 \mathrm{E}+00$ & $1.52 \mathrm{E}-06$ \\
\hline 9 & 42.34 & 0.024 & $-9.87 \mathrm{E}-05$ & 0.000 & $9.74 \mathrm{E}-09$ & $1.00 \mathrm{E}+00$ & $2.23 \mathrm{E}-14$ \\
\hline 10 & 43.61 & 0.023 & $5.38 \mathrm{E}+00$ & 0.009 & $2.90 \mathrm{E}+01$ & $1.00 \mathrm{E}+00$ & $6.62 \mathrm{E}-05$ \\
\hline sum & & & & & $4.37 \mathrm{E}+05$ & & $1.00 \mathrm{E}+00$ \\
\hline
\end{tabular}

Table 5. Participation factor calculation ROT X direction

\begin{tabular}{ccccccc}
\hline MODE & FREQUENCY PERIOD & $\begin{array}{c}\text { PARTIC. } \\
\text { FACTOR }\end{array}$ & RATIO & $\begin{array}{c}\text { EFFECTIV } \\
\text { E MASS }\end{array}$ & $\begin{array}{c}\text { CUMULATI } \\
\text { VE MASS } \\
\text { FRACTION }\end{array}$ \\
\hline 1 & 6.61 & 0.151 & $1.81 \mathrm{E}+03$ & 0.991 & $3.29 \mathrm{E}+06$ & $4.64 \mathrm{E}-01$ \\
\hline 2 & 8.68 & 0.115 & $-8.64 \mathrm{E}+00$ & 0.005 & 74.5847 & $4.64 \mathrm{E}-01$ \\
\hline 3 & 11.27 & 0.089 & $3.87 \mathrm{E}+01$ & 0.021 & $1.49 \mathrm{E}+03$ & $4.64 \mathrm{E}-01$ \\
\hline 4 & 18.32 & 0.055 & $-1.83 \mathrm{E}+03$ & 1.000 & 3353000 & $9.37 \mathrm{E}-01$ \\
\hline 5 & 18.97 & 0.053 & $-5.87 \mathrm{E}+02$ & 0.320 & $3.44 \mathrm{E}+05$ & $9.85 \mathrm{E}-01$ \\
\hline 6 & 20.51 & 0.049 & $-8.80 \mathrm{E}+01$ & 0.048 & $7.75 \mathrm{E}+03$ & $9.86 \mathrm{E}-01$ \\
\hline 7 & 26.22 & 0.038 & $8.38 \mathrm{E}+01$ & 0.046 & $7.03 \mathrm{E}+03$ & $9.87 \mathrm{E}-01$ \\
\hline 8 & 26.91 & 0.037 & $6.29 \mathrm{E}+01$ & 0.034 & $3.96 \mathrm{E}+03$ & $9.88 \mathrm{E}-01$ \\
\hline 9 & 42.34 & 0.024 & $-2.34 \mathrm{E}+02$ & 0.128 & $5.46 \mathrm{E}+04$ & $9.95 \mathrm{E}-01$ \\
\hline 10 & 43.61 & 0.023 & $-1.80 \mathrm{E}+02$ & 0.099 & $3.26 \mathrm{E}+04$ & $1.00 \mathrm{E}+00$ \\
\hline sum & & & & & $7.10 \mathrm{E}+06$ & \\
\hline
\end{tabular}

Table 6. Participation factor calculation ROT Y direction

\begin{tabular}{ccccccc}
\hline MODE & FREQUENCY PERIOD & $\begin{array}{c}\text { PARTIC. } \\
\text { FACTOR }\end{array}$ & RATIO & $\begin{array}{c}\text { EFFECTIV } \\
\text { E MASS }\end{array}$ & $\begin{array}{c}\text { CUMULATI } \\
\text { VE MASS } \\
\text { FRACTION }\end{array}$ \\
\hline 1 & 6.61 & 0.151 & $-3.60 \mathrm{E}+03$ & 1.000 & $1.30 \mathrm{E}+07$ & $4.66 \mathrm{E}-01$ \\
\hline 2 & 8.68 & 0.115 & $1.74 \mathrm{E}+03$ & 0.482 & 3011890 & $5.74 \mathrm{E}-01$ \\
\hline 3 & 11.27 & 0.089 & $-2.52 \mathrm{E}+03$ & 0.698 & $6.33 \mathrm{E}+06$ & $8.02 \mathrm{E}-01$ \\
\hline 4 & 18.32 & 0.055 & $-3.34 \mathrm{E}+01$ & 0.009 & 1116.37 & $8.02 \mathrm{E}-01$ \\
\hline 5 & 18.97 & 0.053 & $-2.15 \mathrm{E}+03$ & 0.596 & $4.61 \mathrm{E}+06$ & $9.67 \mathrm{E}-01$ \\
\hline 6 & 20.51 & 0.049 & $6.29 \mathrm{E}+02$ & 0.175 & $3.96 \mathrm{E}+05$ & $9.81 \mathrm{E}-01$ \\
\hline 7 & 26.22 & 0.038 & $1.38 \mathrm{E}+02$ & 0.038 & $1.90 \mathrm{E}+04$ & $9.82 \mathrm{E}-01$ \\
\hline 8 & 26.91 & 0.037 & $-7.03 \mathrm{E}+02$ & 0.195 & $4.94 \mathrm{E}+05$ & $1.00 \mathrm{E}+00$ \\
\hline 9 & 42.34 & 0.024 & $9.27 \mathrm{E}+00$ & 0.003 & $8.60 \mathrm{E}+01$ & $1.00 \mathrm{E}+00$ \\
\hline 10 & 43.61 & 0.023 & $-8.73 \mathrm{E}+01$ & 0.024 & $7.61 \mathrm{E}+03$ & $1.00 \mathrm{E}+00$ \\
\hline sum & & & & & $2.78 \mathrm{E}+07$ & \\
\hline
\end{tabular}

Table 7. Participation factor calculation ROT Z direction

\begin{tabular}{ccccccc}
\hline MODE FREQUENCY PERIOD & $\begin{array}{c}\text { PARTIC. } \\
\text { FACTOR }\end{array}$ & RATIO & $\begin{array}{c}\text { EFFECTIV } \\
\text { E MASS }\end{array}$ & $\begin{array}{c}\text { CUMULATI } \\
\text { VE MASS } \\
\text { FRACTION }\end{array}$ \\
\hline 1 & 6.61 & 0.151 & $1.06 \mathrm{E}-04$ & 0.000 & $1.12 \mathrm{E}-08$ & $4.18 \mathrm{E}-16$ \\
\hline 2 & 8.68 & 0.115 & $-1.85 \mathrm{E}+03$ & 0.447 & 3425700 & $1.28 \mathrm{E}-01$ \\
\hline 3 & 11.27 & 0.089 & $-8.39 \mathrm{E}-05$ & 0.000 & $7.04 \mathrm{E}-09$ & $1.28 \mathrm{E}-01$ \\
\hline 4 & 18.32 & 0.055 & $4.14 \mathrm{E}+03$ & 1.000 & 17107200 & $7.65 \mathrm{E}-01$ \\
\hline 5 & 18.97 & 0.053 & $7.26 \mathrm{E}-04$ & 0.000 & $5.27 \mathrm{E}-07$ & $7.65 \mathrm{E}-01$ \\
\hline 6 & 20.51 & 0.049 & $1.82 \mathrm{E}+03$ & 0.440 & $3.31 \mathrm{E}+06$ & $8.88 \mathrm{E}-01$ \\
\hline 7 & 26.22 & 0.038 & $-1.60 \mathrm{E}+03$ & 0.387 & $2.56 \mathrm{E}+06$ & $9.83 \mathrm{E}-01$ \\
\hline 8 & 26.91 & 0.037 & $-1.40 \mathrm{E}-03$ & 0.000 & $1.95 \mathrm{E}-06$ & $9.83 \mathrm{E}-01$ \\
\hline 9 & 42.34 & 0.024 & $6.69 \mathrm{E}+02$ & 0.162 & $4.47 \mathrm{E}+05$ & $1.00 \mathrm{E}+00$ \\
\hline 10 & 43.61 & 0.023 & $1.26 \mathrm{E}-02$ & 0.000 & $1.58 \mathrm{E}-04$ & $1.00 \mathrm{E}+00$ \\
\hline sum & & & & & $2.68 \mathrm{E}+07$ & \\
\hline & & & & & & \\
\hline
\end{tabular}

5.2 ANSYS - natural frequencies for lower "E"

Table 8. Participation factor calculation $\mathrm{x}$ direction

\begin{tabular}{cccccccc}
\hline MODE FREQUENCY PERIOD & $\begin{array}{c}\text { PARTIC. } \\
\text { FACTOR }\end{array}$ & RATIO & $\begin{array}{c}\text { EFFECTIVE } \\
\text { MASS }\end{array}$ & $\begin{array}{c}\text { CUMULATIVE } \\
\text { MASS } \\
\text { FRACTION }\end{array}$ & $\begin{array}{c}\text { RATIO } \\
\text { EFF.MASS } \\
\text { TO TOTAL } \\
\text { MASS }\end{array}$ \\
\hline 1 & 6.41 & 0.156 & $-1.18 \mathrm{E}-05$ & 0.000 & $1.39 \mathrm{E}-10$ & $3.18 \mathrm{E}-16$ & $3.18 \mathrm{E}-16$ \\
\hline 2 & 8.35 & 0.120 & $6.13 \mathrm{E}+02$ & 1.000 & 375775 & $8.60 \mathrm{E}-01$ & 0.85942 \\
\hline 3 & 10.85 & 0.092 & $9.69 \mathrm{E}-06$ & 0.000 & $9.39 \mathrm{E}-11$ & $8.60 \mathrm{E}-01$ & $2.15 \mathrm{E}-16$ \\
\hline 4 & 17.72 & 0.056 & $-6.97 \mathrm{E}+00$ & 0.011 & 48.6029 & $8.60 \mathrm{E}-01$ & $1.11 \mathrm{E}-04$ \\
\hline 5 & 18.55 & 0.054 & $-9.33 \mathrm{E}-06$ & 0.000 & $8.70 \mathrm{E}-11$ & $8.60 \mathrm{E}-01$ & $1.99 \mathrm{E}-16$ \\
\hline 6 & 19.79 & 0.051 & $2.43 \mathrm{E}+02$ & 0.397 & $5.92 \mathrm{E}+04$ & $9.95 \mathrm{E}-01$ & $1.35 \mathrm{E}-01$ \\
\hline 7 & 24.87 & 0.040 & $4.62 \mathrm{E}+01$ & 0.075 & $2.14 \mathrm{E}+03$ & $1.00 \mathrm{E}+00$ & $4.89 \mathrm{E}-03$ \\
\hline 8 & 25.47 & 0.039 & $4.62 \mathrm{E}-05$ & 0.000 & $2.13 \mathrm{E}-09$ & $1.00 \mathrm{E}+00$ & $4.88 \mathrm{E}-15$ \\
\hline 9 & 39.68 & 0.025 & $3.68 \mathrm{E}+00$ & 0.006 & $1.35 \mathrm{E}+01$ & $1.00 \mathrm{E}+00$ & $3.09 \mathrm{E}-05$ \\
\hline 10 & 40.50 & 0.025 & $1.05 \mathrm{E}-04$ & 0.000 & $1.11 \mathrm{E}-08$ & $1.00 \mathrm{E}+00$ & $2.53 \mathrm{E}-14$ \\
\hline sum & & & & & $4.37 \mathrm{E}+05$ & & $1.00 \mathrm{E}+00$ \\
\hline
\end{tabular}

Table 9. Participation factor calculation Y direction

\begin{tabular}{cccccccc}
\hline MODE FREQUENCY PERIOD & $\begin{array}{c}\text { PARTIC. } \\
\text { FACTOR }\end{array}$ & RATIO & $\begin{array}{c}\text { EFFECTIVE } \\
\text { MASS }\end{array}$ & $\begin{array}{c}\text { CUMULATIVE } \\
\text { MASS } \\
\text { FRACTION }\end{array}$ & $\begin{array}{c}\text { RATIO } \\
\text { EFF.MASS } \\
\text { TO TOTAL } \\
\text { MASS }\end{array}$ \\
\hline 1 & 6.41 & 0.156 & $1.21 \mathrm{E}-05$ & 0.000 & $1.46 \mathrm{E}-10$ & $3.35 \mathrm{E}-16$ & $3.35 \mathrm{E}-16$ \\
\hline 2 & 8.35 & 0.120 & $2.29 \mathrm{E}+00$ & 0.004 & 5.2439 & $1.20 \mathrm{E}-05$ & $1.1993 \mathrm{E}-05$ \\
\hline 3 & 10.85 & 0.092 & $-3.40 \mathrm{E}-06$ & 0.000 & $1.16 \mathrm{E}-11$ & $1.20 \mathrm{E}-05$ & $2.64 \mathrm{E}-17$ \\
\hline 4 & 17.72 & 0.056 & $6.53 \mathrm{E}+02$ & 1.000 & 426789 & $9.78 \mathrm{E}-01$ & $9.76 \mathrm{E}-01$ \\
\hline 5 & 18.55 & 0.054 & $9.69 \mathrm{E}-05$ & 0.000 & $9.38 \mathrm{E}-09$ & $9.78 \mathrm{E}-01$ & $2.15 \mathrm{E}-14$ \\
\hline 6 & 19.79 & 0.051 & $1.70 \mathrm{E}+01$ & 0.026 & $2.91 \mathrm{E}+02$ & $9.79 \mathrm{E}-01$ & $6.65 \mathrm{E}-04$ \\
\hline 7 & 24.87 & 0.040 & $-2.96 \mathrm{E}+01$ & 0.045 & $8.77 \mathrm{E}+02$ & $9.81 \mathrm{E}-01$ & $2.00 \mathrm{E}-03$ \\
\hline 8 & 25.47 & 0.039 & $1.71 \mathrm{E}-05$ & 0.000 & $2.91 \mathrm{E}-10$ & $9.81 \mathrm{E}-01$ & $6.66 \mathrm{E}-16$ \\
\hline 9 & 39.68 & 0.025 & $9.16 \mathrm{E}+01$ & 0.140 & $8.39 \mathrm{E}+03$ & $1.00 \mathrm{E}+00$ & $1.92 \mathrm{E}-02$ \\
\hline 10 & 40.50 & 0.025 & $2.49 \mathrm{E}-03$ & 0.000 & $6.21 \mathrm{E}-06$ & $1.00 \mathrm{E}+00$ & $1.42 \mathrm{E}-11$ \\
\hline sum & & & & & $4.36 \mathrm{E}+05$ & & $9.98 \mathrm{E}-01$ \\
\hline & & & & & & & \\
\hline
\end{tabular}

Table 10. Participation factor calculation $\mathrm{z}$ direction

\begin{tabular}{cccccccc}
\hline MODE RREQUENCY PERIOD & $\begin{array}{c}\text { PARTIC. } \\
\text { FACTOR }\end{array}$ & RATIO & $\begin{array}{c}\text { EFFECTIVE } \\
\text { MASS }\end{array}$ & $\begin{array}{c}\text { CUMULATIVE } \\
\text { MASS } \\
\text { FRACTION }\end{array}$ & $\begin{array}{c}\text { RATIO } \\
\text { EFF.MASS } \\
\text { TO TOTAL } \\
\text { MASS }\end{array}$ \\
\hline 1 & 6.41 & 0.156 & $5.75 \mathrm{E}+02$ & 1.000 & $3.30 \mathrm{E}+05$ & $7.56 \mathrm{E}-01$ & $7.56 \mathrm{E}-01$ \\
\hline 2 & 8.35 & 0.120 & $1.23 \mathrm{E}-05$ & 0.000 & $1.52 \mathrm{E}-10$ & $7.56 \mathrm{E}-01$ & $3.465 \mathrm{E}-16$ \\
\hline 3 & 10.85 & 0.092 & $3.74 \mathrm{E}+01$ & 0.065 & $1.40 \mathrm{E}+03$ & $7.59 \mathrm{E}-01$ & $3.20 \mathrm{E}-03$ \\
\hline 4 & 17.72 & 0.056 & $-5.82 \mathrm{E}-05$ & 0.000 & $3.38 \mathrm{E}-09$ & $7.59 \mathrm{E}-01$ & $7.74 \mathrm{E}-15$ \\
\hline 5 & 18.55 & 0.054 & $3.24 \mathrm{E}+02$ & 0.564 & $1.05 \mathrm{E}+05$ & $1.00 \mathrm{E}+00$ & $2.41 \mathrm{E}-01$ \\
\hline 6 & 19.79 & 0.051 & $5.21 \mathrm{E}-06$ & 0.000 & $2.71 \mathrm{E}-11$ & $1.00 \mathrm{E}+00$ & $6.21 \mathrm{E}-17$ \\
\hline 7 & 24.87 & 0.040 & $7.90 \mathrm{E}-06$ & 0.000 & $6.24 \mathrm{E}-11$ & $1.00 \mathrm{E}+00$ & $1.43 \mathrm{E}-16$ \\
\hline 8 & 25.47 & 0.039 & $-2.75 \mathrm{E}+00$ & 0.005 & $7.59 \mathrm{E}+00$ & $1.00 \mathrm{E}+00$ & $1.74 \mathrm{E}-05$ \\
\hline 9 & 39.68 & 0.025 & $-1.81 \mathrm{E}-04$ & 0.000 & $3.29 \mathrm{E}-08$ & $1.00 \mathrm{E}+00$ & $7.52 \mathrm{E}-14$ \\
\hline 10 & 40.50 & 0.025 & $6.65 \mathrm{E}+00$ & 0.012 & $4.42 \mathrm{E}+01$ & $1.00 \mathrm{E}+00$ & $1.01 \mathrm{E}-04$ \\
\hline sum & & & & & $4.37 \mathrm{E}+05$ & & $1.00 \mathrm{E}+00$ \\
\hline
\end{tabular}

Table 11. Participation factor calculation ROTX direction

\begin{tabular}{ccccccc}
\hline MODE FREQUENCY PERIOD & $\begin{array}{c}\text { PARTIC. } \\
\text { FACTOR }\end{array}$ & RATIO & $\begin{array}{c}\text { EFFCTIVE } \\
\text { MASS }\end{array}$ & $\begin{array}{c}\text { CUMULATIVE } \\
\text { MASS } \\
\text { RACTION }\end{array}$ \\
\hline 1 & 6.41 & 0.156 & $1.82 \mathrm{E}+03$ & 0.995 & $3.31 \mathrm{E}+06$ & $4.67 \mathrm{E}-01$ \\
\hline 2 & 8.35 & 0.120 & $-6.41 \mathrm{E}+00$ & 0.004 & 41.1117 & $4.67 \mathrm{E}-01$ \\
\hline 3 & 10.85 & 0.092 & $3.57 \mathrm{E}+01$ & 0.020 & $1.27 \mathrm{E}+03$ & $4.67 \mathrm{E}-01$ \\
\hline 4 & 17.72 & 0.056 & $-1.83 \mathrm{E}+03$ & 1.000 & 3346020 & $9.39 \mathrm{E}-01$ \\
\hline 5 & 18.55 & 0.054 & $-5.62 \mathrm{E}+02$ & 0.307 & $3.16 \mathrm{E}+05$ & $9.83 \mathrm{E}-01$ \\
\hline 6 & 19.79 & 0.051 & $-4.77 \mathrm{E}+01$ & 0.026 & $2.28 \mathrm{E}+03$ & $9.84 \mathrm{E}-01$ \\
\hline 7 & 24.87 & 0.040 & $8.29 \mathrm{E}+01$ & 0.045 & $6.87 \mathrm{E}+03$ & $9.84 \mathrm{E}-01$ \\
\hline 8 & 25.47 & 0.039 & $7.22 \mathrm{E}+01$ & 0.039 & $5.21 \mathrm{E}+03$ & $9.85 \mathrm{E}-01$ \\
\hline 9 & 39.68 & 0.025 & $-2.56 \mathrm{E}+02$ & 0.140 & $6.58 \mathrm{E}+04$ & $9.94 \mathrm{E}-01$ \\
\hline 10 & 40.50 & 0.025 & $-1.98 \mathrm{E}+02$ & 0.108 & $3.90 \mathrm{E}+04$ & $1.00 \mathrm{E}+00$ \\
\hline sum & & & & & $7.10 \mathrm{E}+06$ & \\
\hline
\end{tabular}

Table 12. Participation factor calculation ROTY direction 


\begin{tabular}{ccccccc}
\hline MODE FREQUENCY PERIOD & $\begin{array}{c}\text { PARTIC. } \\
\text { FACTOR }\end{array}$ & RATIO & $\begin{array}{c}\text { EFECTIVE } \\
\text { MASS }\end{array}$ & $\begin{array}{c}\text { CUMULATIVE } \\
\text { MASS } \\
\text { FRACTION }\end{array}$ \\
\hline 1 & 6.41 & 0.156 & $-3.58 \mathrm{E}+03$ & 1.000 & $1.28 \mathrm{E}+07$ & $4.61 \mathrm{E}-01$ \\
\hline 2 & 8.35 & 0.120 & $1.72 \mathrm{E}+03$ & 0.479 & 2946070 & $5.67 \mathrm{E}-01$ \\
\hline 3 & 10.85 & 0.092 & $-2.47 \mathrm{E}+03$ & 0.691 & $6.12 \mathrm{E}+06$ & $7.87 \mathrm{E}-01$ \\
\hline 4 & 17.72 & 0.056 & $-1.95 \mathrm{E}+01$ & 0.005 & 381.031 & $7.87 \mathrm{E}-01$ \\
\hline 5 & 18.55 & 0.054 & $-2.20 \mathrm{E}+03$ & 0.615 & $4.86 \mathrm{E}+06$ & $9.61 \mathrm{E}-01$ \\
\hline 6 & 19.79 & 0.051 & $6.81 \mathrm{E}+02$ & 0.190 & $4.64 \mathrm{E}+05$ & $9.78 \mathrm{E}-01$ \\
\hline 7 & 24.87 & 0.040 & $1.29 \mathrm{E}+02$ & 0.036 & $1.68 \mathrm{E}+04$ & $9.78 \mathrm{E}-01$ \\
\hline 8 & 25.47 & 0.039 & $-7.68 \mathrm{E}+02$ & 0.214 & $5.89 \mathrm{E}+05$ & $1.00 \mathrm{E}+00$ \\
\hline 9 & 39.68 & 0.025 & $1.03 \mathrm{E}+01$ & 0.003 & $1.06 \mathrm{E}+02$ & $1.00 \mathrm{E}+00$ \\
\hline 10 & 40.50 & 0.025 & $-1.03 \mathrm{E}+02$ & 0.029 & $1.06 \mathrm{E}+04$ & $1.00 \mathrm{E}+00$ \\
\hline sum & & & & & $2.78 \mathrm{E}+07$ & \\
\hline
\end{tabular}

Table 13. Participation factor calculation ROTZ direction

\begin{tabular}{ccccccc}
\hline MODE RREQUENCY PERIOD & $\begin{array}{c}\text { PARTIC. } \\
\text { FACTOR }\end{array}$ & RATIO & $\begin{array}{c}\text { EFFECTIVE } \\
\text { MASS }\end{array}$ & $\begin{array}{c}\text { CUMULATIVE } \\
\text { MASS } \\
\text { FRACTION }\end{array}$ \\
\hline 1 & 6.41 & 0.156 & $1.15 \mathrm{E}-04$ & 0.000 & $1.31 \mathrm{E}-08$ & $4.90 \mathrm{E}-16$ \\
\hline 2 & 8.35 & 0.120 & $-1.87 \mathrm{E}+03$ & 0.449 & 3492700 & $1.30 \mathrm{E}-01$ \\
\hline 3 & 10.85 & 0.092 & $-8.72 \mathrm{E}-05$ & 0.000 & $7.61 \mathrm{E}-09$ & $1.30 \mathrm{E}-01$ \\
\hline 4 & 17.72 & 0.056 & $4.16 \mathrm{E}+03$ & 1.000 & 17306000 & $7.75 \mathrm{E}-01$ \\
\hline 5 & 18.55 & 0.054 & $6.29 \mathrm{E}-04$ & 0.000 & $3.95 \mathrm{E}-07$ & $7.75 \mathrm{E}-01$ \\
\hline 6 & 19.79 & 0.051 & $1.59 \mathrm{E}+03$ & 0.382 & $2.53 \mathrm{E}+06$ & $8.70 \mathrm{E}-01$ \\
\hline 7 & 24.87 & 0.040 & $-1.72 \mathrm{E}+03$ & 0.414 & $2.97 \mathrm{E}+06$ & $9.80 \mathrm{E}-01$ \\
\hline 8 & 25.47 & 0.039 & $-1.56 \mathrm{E}-03$ & 0.000 & $2.43 \mathrm{E}-06$ & $9.80 \mathrm{E}-01$ \\
\hline 9 & 39.68 & 0.025 & $7.31 \mathrm{E}+02$ & 0.176 & $5.34 \mathrm{E}+05$ & $1.00 \mathrm{E}+00$ \\
\hline 10 & 40.50 & 0.025 & $2.01 \mathrm{E}-02$ & 0.000 & $4.02 \mathrm{E}-04$ & $1.00 \mathrm{E}+00$ \\
\hline sum & & & & & $2.68 \mathrm{E}+07$ & \\
\hline
\end{tabular}

Upper E value:

The natural frequency along the rotor direction(X-dir)

$=8.67566 \mathrm{~Hz} \quad:$ 2nd Mode

The natural frequency across the rotor direction (Z-dir)

$=6.61252 \mathrm{~Hz} \quad: 1 \mathrm{st}$ Mode

The natural frequency in vertical direction $(\mathrm{Y}$-dir) $=18.3195$ $\mathrm{Hz} \quad$ : 4th Mode

Lower E value:

The natural frequency along the rotor direction(X-dir) = $8.35271 \mathrm{~Hz} \quad$ : 2nd Mode

The natural frequency across the rotor direction (Z-dir) = $6.40527 \mathrm{~Hz}$ : : 1st Mode

The natural frequency in vertical direction (Y-dir) $=17.718$ $\mathrm{Hz} \quad$ : 4th Mode

The operating frequency of the Fan and motor $\quad=1490 / 60$ $=24.83 \mathrm{~Hz}$

The natural frequencies are away from the frequency range,

Fan and Motor: $19.88 \mathrm{~Hz}(0.8 * 24.83)$ To $29.80 \mathrm{~Hz}(1.2 * 24.83)$ Also, $42.21 \mathrm{~Hz}(0.85 * 2 * 24.83)$ to $57.10 \mathrm{~Hz}(1.15 * 2 * 24.83)$
5.3 ANSYS - mode shape plots for upper young's modulus

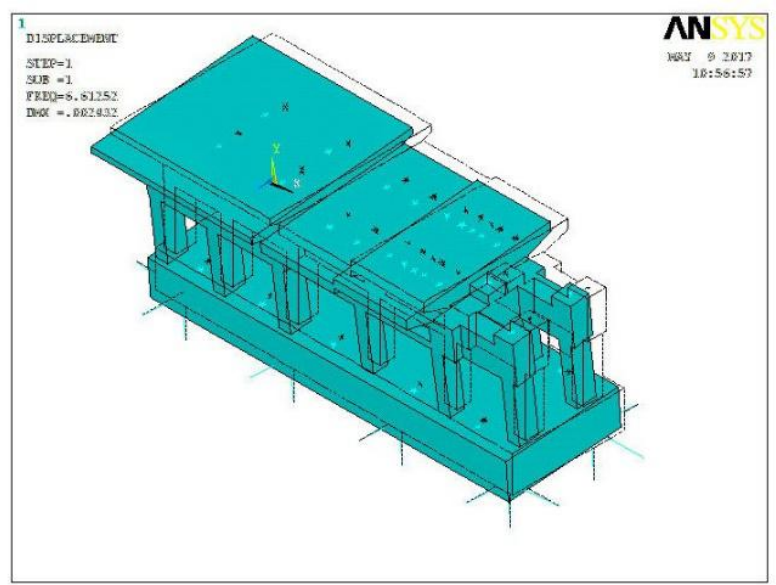

Figure 5. Mode no. 1 : Deformation plot

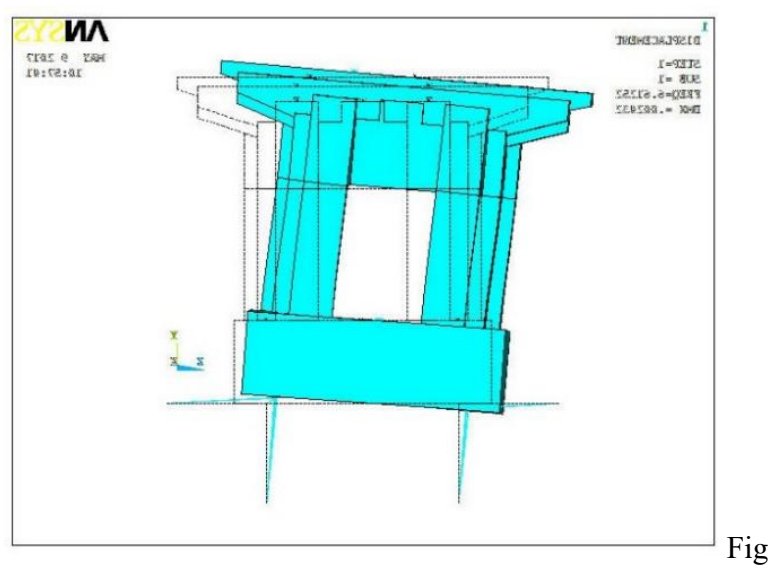

ure 6. Mode no.1: Deformation plot - side view

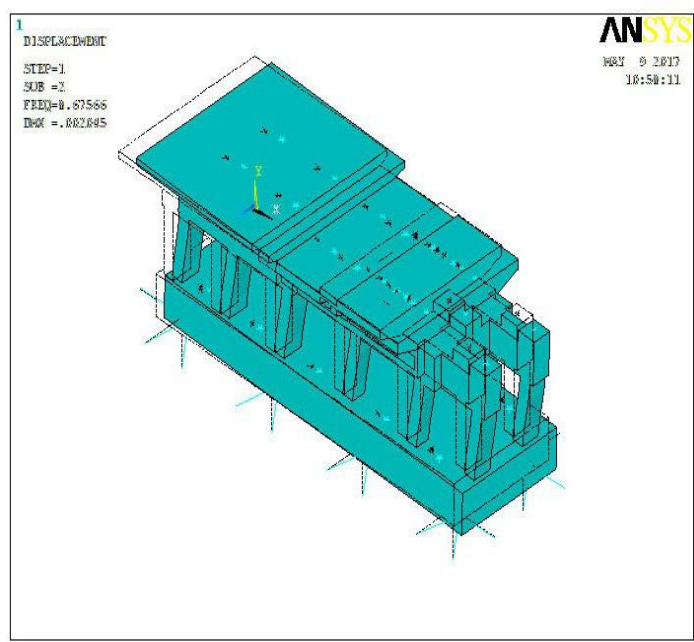

Figure 7. Mode no. 2 : Deformation plot 


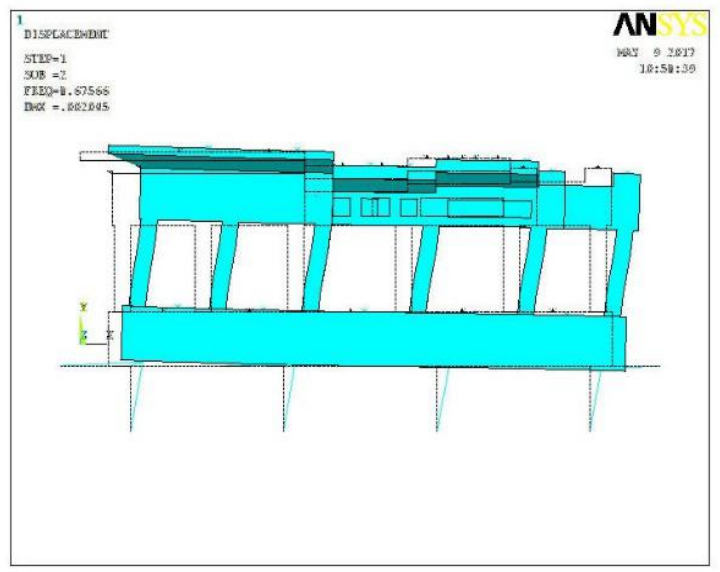

Figure 8. Mode no. 2 : Deformation plot - side view

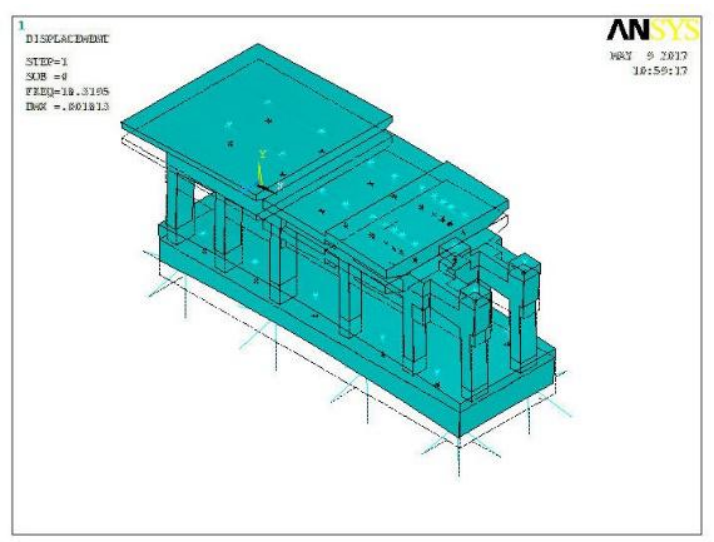

Figure. 9. Mode no. 4 Deformation plot

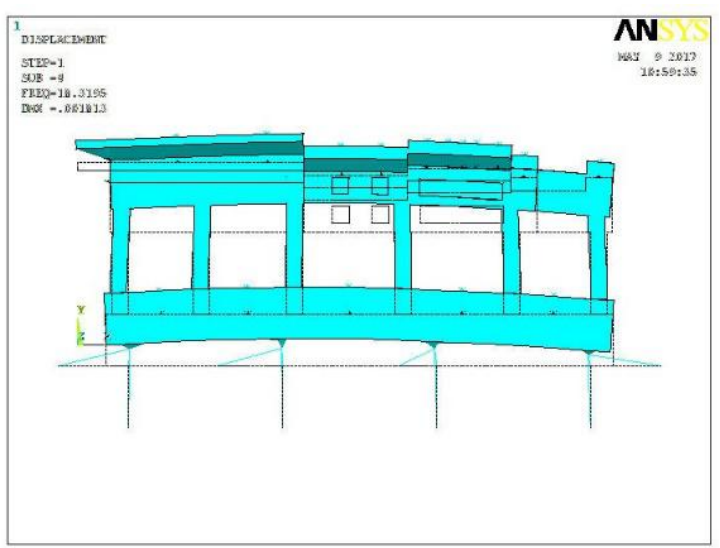

Figure 10. Mode no. 4 deformation plot - side view
5.4 ANSYS - mode shape plots for lower young's modulus

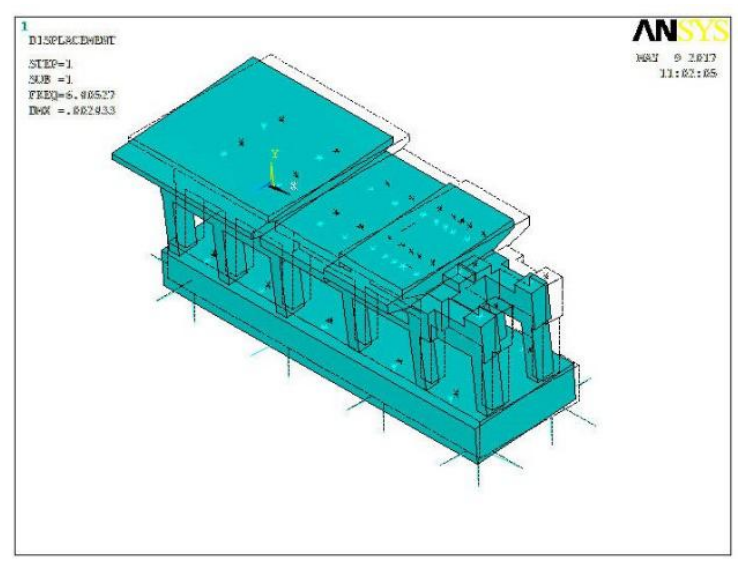

Figure 11. Mode no. 1: Deformation plot

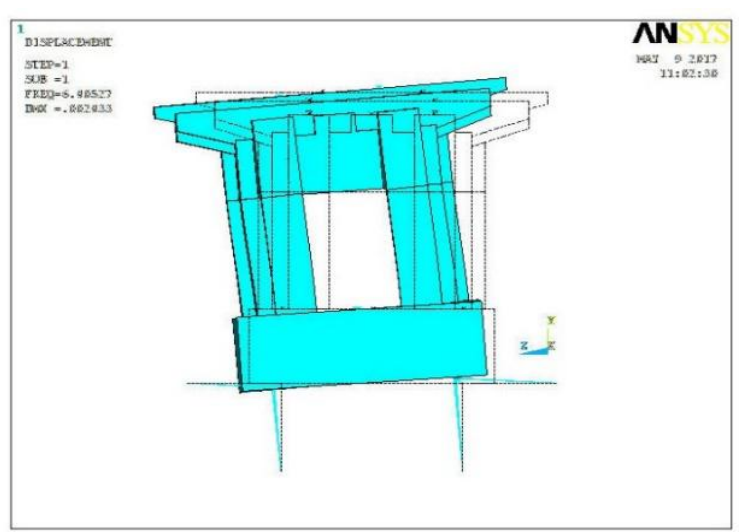

Figure 12. Mode no.1: Deformation plot - side view

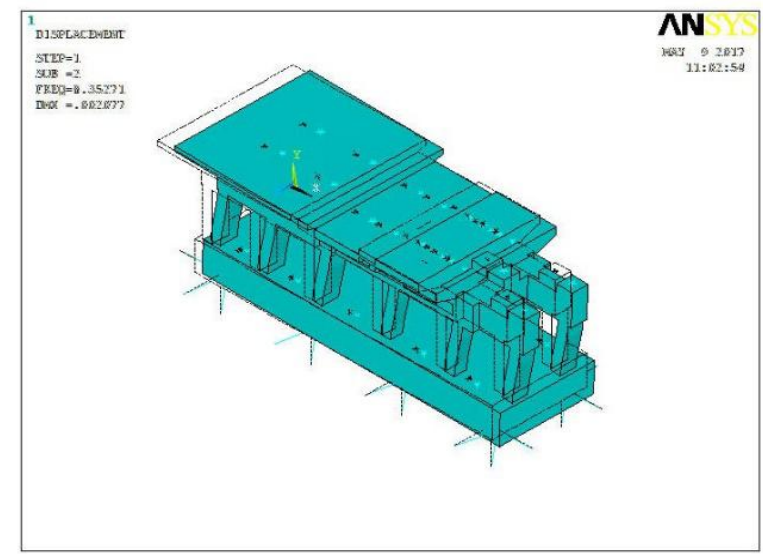

Figure 13. Mode no. 2 : deformation plot 


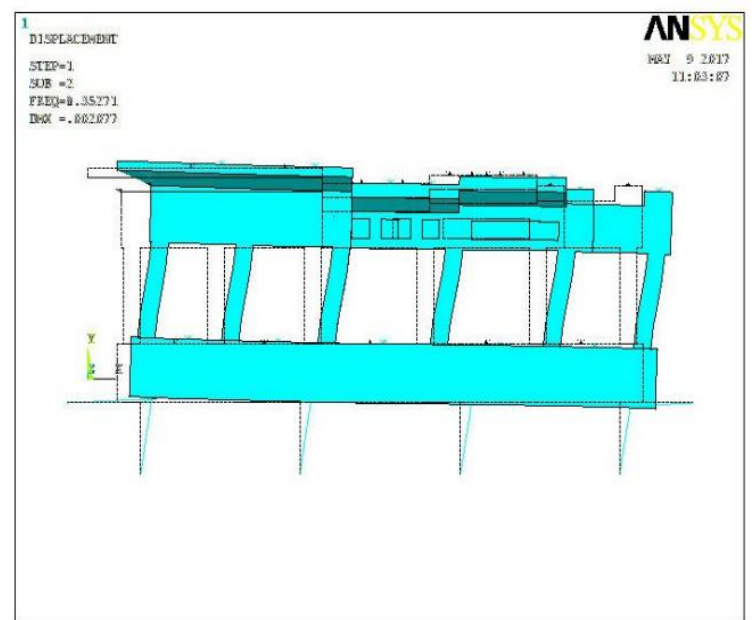

Figure 14. Mode no. 2 : Deformation plot - side view

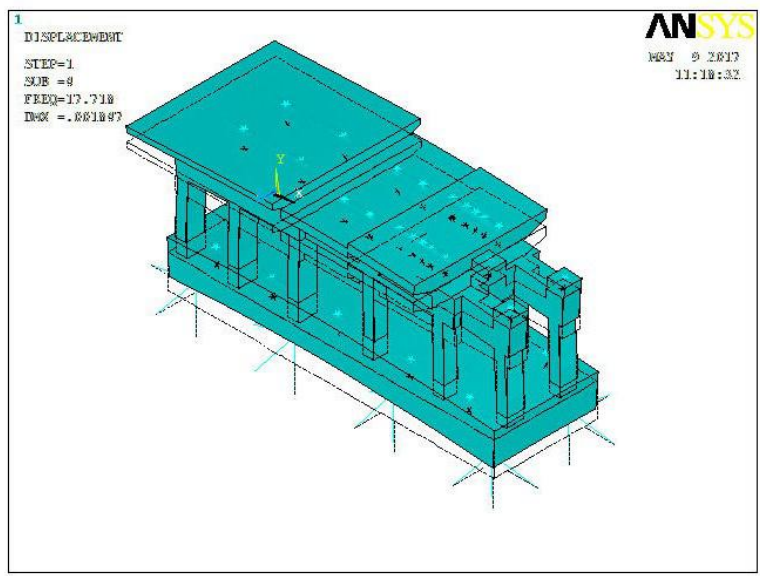

Figure 15. Mode no. 4 Deformation plot

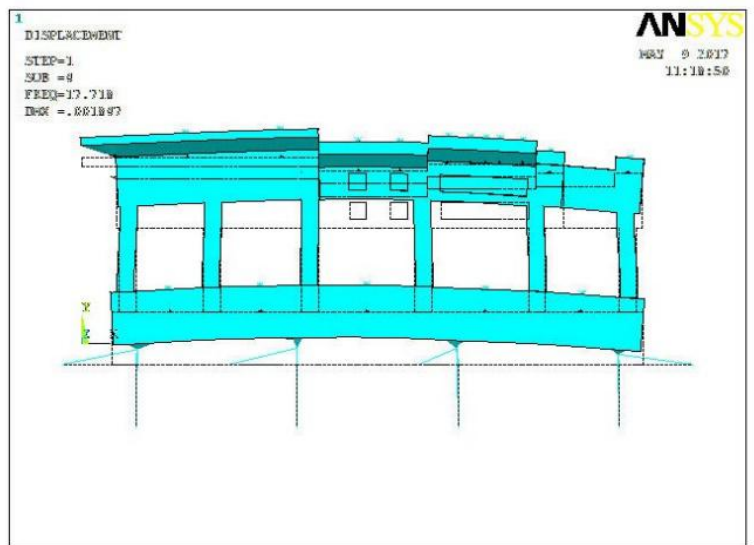

Figure 16. Mode no. 4 deformation plot - side view

\subsection{ANSYS - amplitude output}

Dynamic Force Calculation

Eccentricity for Fan and Motor Rotor

Motor speed $=1490 \mathrm{RPM}=156 \mathrm{rad} / \mathrm{sec}=24.83 \mathrm{~Hz}$
Balance quality grade $=\mathrm{G} 6.3$ (as per ISO :1940$1: 2003)$

The dynamic forces are estimated as per the machinebalanced grade. The machine-balanced grade is 6.3 . The next lower grade is G16.

Soe ${ }_{\omega}=16 \mathrm{~mm} / \mathrm{sec}$

Eccentricity for motor rotor, $\mathrm{e}=0.1026 \mathrm{~mm}$

Table 14. Unbalanced Force Table

\begin{tabular}{ccc}
\hline \multirow{2}{*}{ Rotor } & Weight, N & \multicolumn{2}{l}{$\begin{array}{l}\text { Unbalanced Force, } \\
\text { mew`2 }\end{array}$} \\
\hline $\begin{array}{c}\text { Fan } \\
\text { Motor }\end{array}$ & 29400 & 7475 \\
& 40000 & 10170 \\
\hline
\end{tabular}

Dynamic Loads Input in Ansys:

Motor:

The centrifugal forces at motor rotor level are transferred onto top deck as below.

Centrifugal force at Motor shaft $\quad=10170 \mathrm{~N}$

Real and Imaginary components of the centrifugal force for motor and fan are considered in the directions as shown on the figures below.

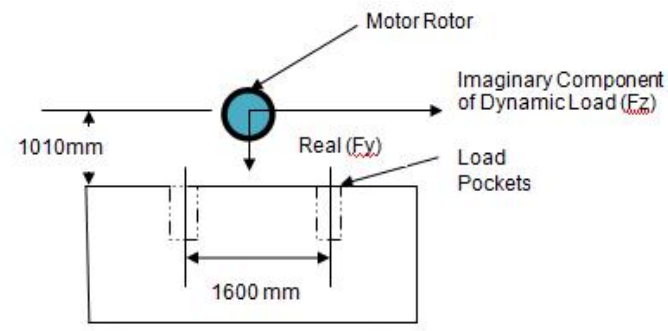

C/s of Motor Base (Section A-A)

Figure 17. C/s of Motor Base (Section A-A)

Number of pockets for motor $\quad=4$

Real component of the centrifugal force on each load pad, Fy $=2542.50 \mathrm{~N}$ (Vertical)

Imaginary component of the dynamic load will have horizontal and a couple on the top deck.

Horizontal load due to imaginary component of the centrifugal force on each pocket, $\mathrm{Fz}=2542.50 \mathrm{~N}(\mathrm{Fz})$

Couple on top deck, $\mathrm{Mx}=10271.7 \mathrm{~N}-\mathrm{m}$

Push Pull (Fy) due to couple on load pads, Fy=3210 N per pocket

\section{Fan:}

The centrifugal forces at fan rotor level are transferred onto top deck as below. 
Centrifugal force at Fan shaft $\quad=7475 \mathrm{~N}$

Number of pockets for Fan $=14$

Real component of the centrifugal force on each load pad, Fy $=534 \mathrm{~N}$ (Vertical)

Imaginary component of the dynamic load will have horizontal and a couple of the top deck.

Horizontal load due to imaginary component of the centrifugal force on each pocket, $\mathrm{Fz}=534 \mathrm{~N}(\mathrm{Fz})$

Couple on top deck, Mx

$=10091.25 \mathrm{~N}-\mathrm{m}$

Push Pull due to couple on load pads, Fy $=720.80 \mathrm{~N}$ per pocket

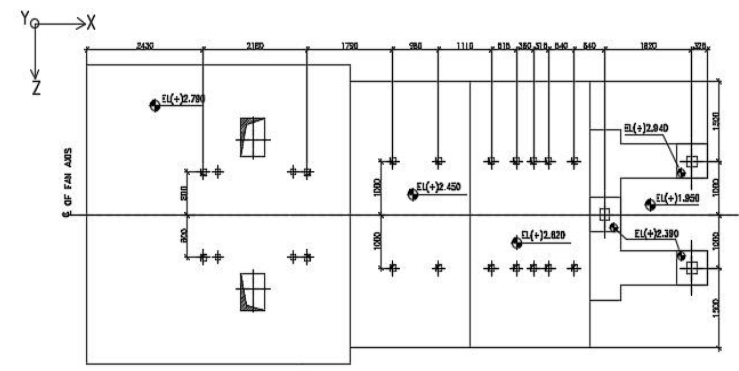

Figure 18. Plan view of PA fan foundation

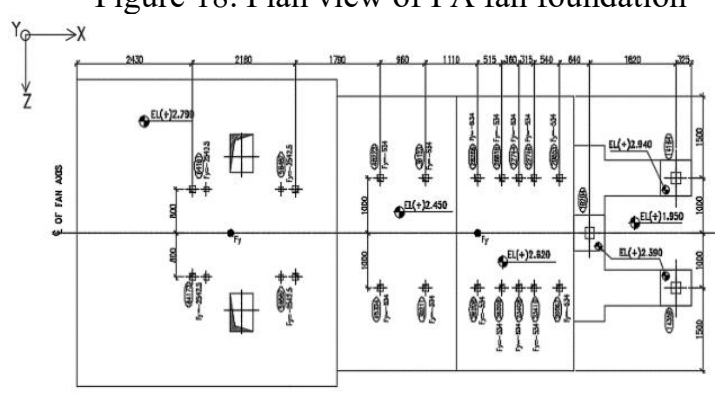

Figure 19. Dynamic Forces - Real Part

(Dynamic Load vertically downward -Y dir.)

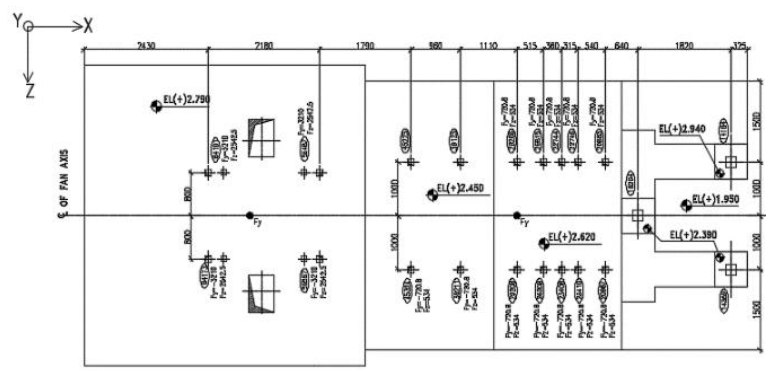

Figure 20. Dynamic Forces - Imaginary Part (Dynamic Load horizontal $+Z$ dir.)

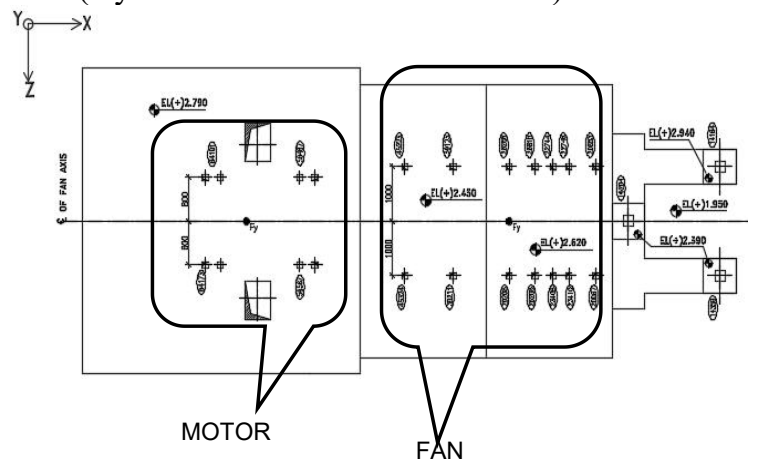

Figure 21. Node numbers at dynamic load points

The above dynamic loads act on the rotor at different phase angles at motor and PA Fan locations. However, as a conservative approach, they are assumed to act simultaneously in the same phase at both locations in the same direction and applied accordingly. The displacement amplitudes are obtained for the above worst condition and are compared with the allowable limits.

Referring to displacement amplitude table, it may be noted that the amplitudes are within the allowable limits

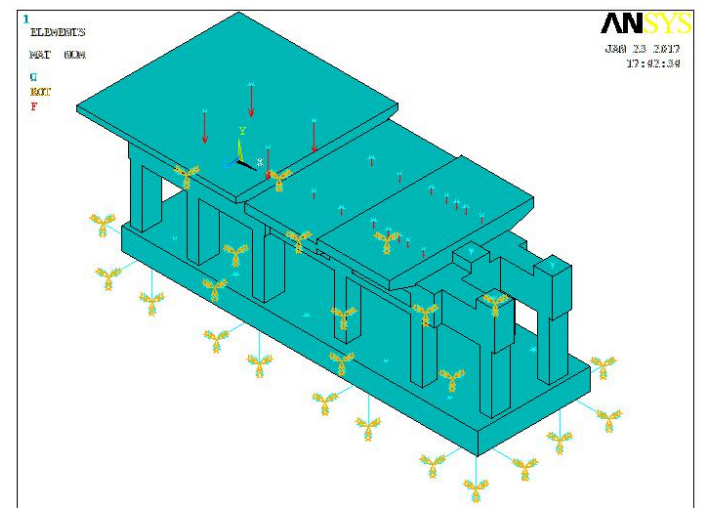

Figure 22. Applied Dynamic Forces

\section{4 displacement amplitudes for operating} frequencies:

Table 15. Upper E Displacement amplitudes for Operating Frequencies

\begin{tabular}{|c|c|c|c|c|c|}
\hline \multirow{2}{*}{$\begin{array}{c}\text { Support Point } \\
\text { at }\end{array}$} & \multirow{2}{*}{ Node } & \multirow{2}{*}{$\begin{array}{l}\text { Frequency } \\
\text { Range }(\mathrm{Hz})\end{array}$} & \multicolumn{3}{|c|}{ Max Amplitude, m } \\
\hline & & & Ux & Uy & Uz \\
\hline \multirow{15}{*}{$\underset{4}{z}$} & 45324 & $24-26$ & $1.11 \mathrm{E}-06$ & $2.63 \mathrm{E}-06$ & $3.35 \mathrm{E}-06$ \\
\hline & 39211 & $24-26$ & $1.14 \mathrm{E}-06$ & $2.77 \mathrm{E}-06$ & $3.63 \mathrm{E}-06$ \\
\hline & 29208 & $24-26$ & $1.05 \mathrm{E}-06$ & $2.87 \mathrm{E}-06$ & $4.36 \mathrm{E}-06$ \\
\hline & 26931 & $24-26$ & $1.09 \mathrm{E}-06$ & $3.16 \mathrm{E}-06$ & $4.70 \mathrm{E}-06$ \\
\hline & 23408 & $24-26$ & $1.11 \mathrm{E}-06$ & $3.38 \mathrm{E}-06$ & $4.92 \mathrm{E}-06$ \\
\hline & 23410 & $24-26$ & 1.12E-06 & $3.57 \mathrm{E}-06$ & $5.09 \mathrm{E}-06$ \\
\hline & 20967 & $24-26$ & $1.15 \mathrm{E}-06$ & $3.85 \mathrm{E}-06$ & $5.35 \mathrm{E}-06$ \\
\hline & 45223 & $24-26$ & $1.61 \mathrm{E}-06$ & $2.71 \mathrm{E}-06$ & $3.35 \mathrm{E}-06$ \\
\hline & 39123 & $24-26$ & $1.61 \mathrm{E}-06$ & $2.87 \mathrm{E}-06$ & $3.63 \mathrm{E}-06$ \\
\hline & 28266 & $24-26$ & $1.42 \mathrm{E}-06$ & $3.47 \mathrm{E}-06$ & 4.37E-06 \\
\hline & 26815 & $24-26$ & $1.45 \mathrm{E}-06$ & $3.91 \mathrm{E}-06$ & $4.72 \mathrm{E}-06$ \\
\hline & 22744 & $24-26$ & $1.46 \mathrm{E}-06$ & $4.20 \mathrm{E}-06$ & 4.94E-06 \\
\hline & 22746 & $24-26$ & $1.48 \mathrm{E}-06$ & 4.44E-06 & $5.11 \mathrm{E}-06$ \\
\hline & 20853 & $24-26$ & $1.50 \mathrm{E}-06$ & $4.81 \mathrm{E}-06$ & $5.37 \mathrm{E}-06$ \\
\hline & Max & & $1.61 \mathrm{E}-06$ & $4.81 \mathrm{E}-06$ & 5.37E-06 \\
\hline \multirow{5}{*}{$\begin{array}{l}\frac{\alpha}{0} \\
\frac{1}{\sigma} \\
\frac{0}{\Sigma}\end{array}$} & 64173 & $24-26$ & $1.01 \mathrm{E}-06$ & $3.06 \mathrm{E}-06$ & $2.71 \mathrm{E}-06$ \\
\hline & 56567 & $24-26$ & $9.14 \mathrm{E}-07$ & $2.26 \mathrm{E}-06$ & 3.17E-06 \\
\hline & 64191 & $24-26$ & $1.46 \mathrm{E}-06$ & $3.34 \mathrm{E}-06$ & $2.71 \mathrm{E}-06$ \\
\hline & 56487 & $24-26$ & $1.31 \mathrm{E}-06$ & 2.29E-06 & 3.17E-06 \\
\hline & $\operatorname{Max}$ & & 1.46E-06 & $3.34 \mathrm{E}-06$ & $3.17 \mathrm{E}-06$ \\
\hline
\end{tabular}


Table 16. Lower E Displacement amplitudes for Operating Frequencies

\begin{tabular}{|c|c|c|c|c|c|}
\hline \multirow{2}{*}{$\begin{array}{c}\text { Support Point } \\
\text { at } \\
\end{array}$} & \multirow{2}{*}{ Node } & \multirow{2}{*}{$\begin{array}{c}\text { Frequency } \\
\text { Range }\end{array}$} & \multicolumn{3}{|c|}{ Max Amplitude, $m$} \\
\hline & & & Ux & Uy & Uz \\
\hline \multirow{15}{*}{$\underset{4}{z}$} & 45324 & $24-26 \mathrm{~Hz}$ & 1.03E-06 & 2.34E-06 & 3.33E-06 \\
\hline & 39211 & $24-26 \mathrm{~Hz}$ & $1.08 \mathrm{E}-06$ & 2.79E-06 & 4.00E-06 \\
\hline & 29208 & $24-26 \mathrm{~Hz}$ & $9.97 \mathrm{E}-07$ & 3.34E-06 & 4.99E-06 \\
\hline & 26931 & $24-26 \mathrm{~Hz}$ & $1.04 \mathrm{E}-06$ & $3.71 \mathrm{E}-06$ & $5.49 \mathrm{E}-06$ \\
\hline & 23408 & $24-26 \mathrm{~Hz}$ & 1.06E-06 & 3.94E-06 & $5.81 \mathrm{E}-06$ \\
\hline & 23410 & $24-26 \mathrm{~Hz}$ & $1.07 \mathrm{E}-06$ & 4.12E-06 & $6.06 \mathrm{E}-06$ \\
\hline & 20967 & $24-26 \mathrm{~Hz}$ & $1.10 \mathrm{E}-06$ & 4.38E-06 & $6.46 \mathrm{E}-06$ \\
\hline & 45223 & $24-26 \mathrm{~Hz}$ & $1.87 \mathrm{E}-06$ & $2.83 \mathrm{E}-06$ & $3.32 \mathrm{E}-06$ \\
\hline & 39123 & $24-26 \mathrm{~Hz}$ & $1.86 \mathrm{E}-06$ & $3.11 \mathrm{E}-06$ & $4.00 \mathrm{E}-06$ \\
\hline & 28266 & $24-26 \mathrm{~Hz}$ & $1.59 \mathrm{E}-06$ & $3.90 \mathrm{E}-06$ & $5.00 \mathrm{E}-06$ \\
\hline & 26815 & $24-26 \mathrm{~Hz}$ & $1.61 \mathrm{E}-06$ & 4.33E-06 & $5.51 \mathrm{E}-06$ \\
\hline & 22744 & $24-26 \mathrm{~Hz}$ & 1.62E-06 & 4.59E-06 & $5.84 \mathrm{E}-06$ \\
\hline & 22746 & $24-26 \mathrm{~Hz}$ & 1.64E-06 & $4.81 \mathrm{E}-06$ & $6.10 \mathrm{E}-06$ \\
\hline & 20853 & $24-26 \mathrm{~Hz}$ & 1.67E-06 & 5.13E-06 & $6.49 \mathrm{E}-06$ \\
\hline & Max & & $1.87 \mathrm{E}-06$ & $5.13 \mathrm{E}-06$ & $6.49 \mathrm{E}-06$ \\
\hline \multirow{5}{*}{$\begin{array}{l}\frac{\alpha}{0} \\
\frac{1}{\circ} \\
\text { 일 }\end{array}$} & 64173 & $24-26 \mathrm{~Hz}$ & 8.77E-07 & 4.36E-06 & $6.79 \mathrm{E}-06$ \\
\hline & 56567 & $24-26 \mathrm{~Hz}$ & 7.91E-07 & $2.88 \mathrm{E}-06$ & $4.66 \mathrm{E}-06$ \\
\hline & 64191 & $24-26 \mathrm{~Hz}$ & $1.67 \mathrm{E}-06$ & $5.66 \mathrm{E}-06$ & $6.77 \mathrm{E}-06$ \\
\hline & 56487 & $24-26 \mathrm{~Hz}$ & $1.46 \mathrm{E}-06$ & 4.11E-06 & 4.65E-06 \\
\hline & $\operatorname{Max}$ & & 1.67E-06 & $5.66 \mathrm{E}-06$ & $6.79 \mathrm{E}-06$ \\
\hline
\end{tabular}

The displacement amplitudes listed in section 5.5 are corresponding the centrifugal force of rotating masses at $1490 \mathrm{Rpm} * 24.83 \mathrm{~Hz}$ ). Displacement amplitudes at other frequencies have to be obtained by following equation.

Displacement amplitude at frequency, $\mathrm{X}=$ (Amplitude at frequency, $\mathrm{X}$ as per section. 5.5/24.83) *Frequency, $\mathrm{X}$

Table 17. The resonant amplitudes during transient stage for Lower E are listed below

\begin{tabular}{|c|c|c|c|c|c|c|c|}
\hline \multirow[t]{2}{*}{$\begin{array}{l}\text { Support } \\
\text { Point at }\end{array}$} & \multirow[t]{2}{*}{ Node } & \multicolumn{3}{|c|}{$\begin{array}{l}\text { Amplitude(in Meters) for dynamic load } \\
\text { corresponding to } 1490 \mathrm{RPM}(24.83 \mathrm{~Hz})\end{array}$} & \multicolumn{3}{|c|}{$\begin{array}{l}\text { Amplitude(in Microns) for dynamic load } \\
\text { corresponding to Respective Natural Frequency (X- } \\
\text { dir 8.5Hz, Y-dir 18Hz, Z-dir 6.5Hz) }\end{array}$} \\
\hline & & $\mathrm{Ux}(8.0-8.5 \mathrm{~Hz}$ & $\mathrm{Uy}(17.5-18 \mathrm{~Hz}$ & $\mathrm{Jz}(6,0,-6.5 \mathrm{~Hz})$ & $\mathrm{Ux}$ & Uy & $\mathrm{U}_{2}$ \\
\hline \multirow{14}{*}{$\frac{2}{4}$} & 45324 & $2.04 \mathrm{E}-06$ & $3.12 \mathrm{E}-05$ & $1.51 \mathrm{E}-04$ & 0.70 & 22.65 & 39.50 \\
\hline & 39211 & $2.02 \mathrm{E}-06$ & $3.06 \mathrm{E}-05$ & $1.50 \mathrm{E}-04$ & 0.69 & 22.17 & 39.22 \\
\hline & 29208 & $1.99 \mathrm{E}-06$ & $2.84 \mathrm{E}-05$ & $1.52 \mathrm{E}-04$ & 0.68 & 20.62 & 39.72 \\
\hline & 26931 & $1.98 \mathrm{E}-06$ & $2.76 \mathrm{E}-05$ & $1.51 \mathrm{E}-04$ & 0.68 & 19.99 & 39.58 \\
\hline & 23408 & $1.98 \mathrm{E}-06$ & $2.68 \mathrm{E}-05$ & $1.51 \mathrm{E}-04$ & 0.68 & 19.40 & 39.46 \\
\hline & 23410 & $1.98 \mathrm{E}-06$ & $2.60 \mathrm{E}-05$ & $1.50 \mathrm{E}-04$ & 0.68 & 18.82 & 39.34 \\
\hline & 20967 & $1.98 \mathrm{E}-06$ & $2.44 \mathrm{E}-05$ & $1.49 \mathrm{E}-04$ & 0.68 & 17.69 & 39.11 \\
\hline & 45223 & $1.77 \mathrm{E}-06$ & $3.53 \mathrm{E}-05$ & $1.51 \mathrm{E}-04$ & 0.61 & 25.60 & 39.50 \\
\hline & 39123 & $1.76 \mathrm{E}-06$ & $3.49 \mathrm{E}-05$ & $1.50 \mathrm{E}-04$ & 0.60 & 25.27 & 39.22 \\
\hline & 28266 & $1.75 \mathrm{E}-06$ & $3.29 \mathrm{E}-05$ & $1.52 \mathrm{E}-04$ & 0.60 & 23.86 & 39.72 \\
\hline & 26815 & $1.75 \mathrm{E}-06$ & $3.22 \mathrm{E}-05$ & $1.51 \mathrm{E}-04$ & 0.60 & 23.37 & 39.58 \\
\hline & 22744 & $1.75 \mathrm{E}-06$ & $3.15 \mathrm{E}-05$ & $1.51 \mathrm{E}-04$ & 0.60 & 22.85 & 39.46 \\
\hline & 22746 & $1.75 \mathrm{E}-06$ & $3.08 \mathrm{E}-05$ & $1.50 \mathrm{E}-04$ & 0.60 & 22.32 & 39.34 \\
\hline & 20853 & $1.75 \mathrm{E}-06$ & $2.93 \mathrm{E}-05$ & $1.49 \mathrm{E}-04$ & 0.60 & 21.22 & 39.11 \\
\hline \multicolumn{2}{|c|}{ MAX. } & $2.04 E-06$ & $3.53 \mathrm{E}-05$ & $1,52 \mathrm{E}-04$ & 0.70 & 25.60 & 39.72 \\
\hline \multirow{4}{*}{$\begin{array}{l}\frac{x}{0} \\
0 \\
0 \\
\\
\Sigma\end{array}$} & 64173 & $2.08 \mathrm{E}-06$ & $2.68 \mathrm{E}-05$ & $1.61 \mathrm{E}-04$ & 0.71 & 19.40 & 42.07 \\
\hline & 56567 & $2.05 \mathrm{E}-06$ & $3.02 \mathrm{E}-05$ & $1.59 \mathrm{E}-04$ & 0.70 & 21.89 & 41.62 \\
\hline & 64191 & $1.86 \mathrm{E}-06$ & $2.92 \mathrm{E}-05$ & $1.61 \mathrm{E}-04$ & 0.64 & 21.20 & 42.07 \\
\hline & 56487 & $1.84 \mathrm{E}-06$ & $3.28 \mathrm{E}-05$ & $1.59 \mathrm{E}-04$ & 0.63 & 23.78 & 41.62 \\
\hline \multicolumn{2}{|c|}{ MAX. } & $2.08 \mathrm{E}-06$ & $3.28 E-05$ & $1.61 \mathrm{E}-04$ & 0.71 & 23.78 & 42.07 \\
\hline
\end{tabular}

Table 18. The resonant amplitudes during transient stage for upper E are listed below

\begin{tabular}{|c|c|c|c|c|c|c|c|}
\hline \multirow[t]{2}{*}{$\begin{array}{l}\text { Support } \\
\text { Point at }\end{array}$} & \multirow[t]{2}{*}{ Node } & \multicolumn{3}{|c|}{$\begin{array}{l}\text { Amplitude(in Meters) for dynamic load } \\
\text { corresponding to } 1490 \mathrm{RPM}(24.83 \mathrm{~Hz})\end{array}$} & \multicolumn{3}{|c|}{$\begin{array}{l}\text { Amplitude(in Microns) for dynamic load } \\
\text { corresponding to Respective Natural Frequency } \\
(X-\text { dir } 9 \mathrm{~Hz}, Y \text {-dir 18.5Hz, Z-dir } 7 \mathrm{~Hz})\end{array}$} \\
\hline & & $\mathrm{Ux}(8.5-9.0 \mathrm{~Hz}$ & $\mathrm{Jy}(18-18.5 \mathrm{~Hz}$ & $\mathrm{Uz}_{2(6.5-7 \mathrm{~Hz})}$ & $U_{x}$ & $U_{Y}$ & $\mathrm{Uz}$ \\
\hline \multirow{14}{*}{$\begin{array}{l}z \\
4 \\
4\end{array}$} & 45324 & $1.84 \mathrm{E}-06$ & $2.72 \mathrm{E}-05$ & $1.36 \mathrm{E}-04$ & 0.67 & 19.71 & 38.43 \\
\hline & 39211 & $1.84 \mathrm{E}-06$ & $2.66 \mathrm{E}-05$ & $1.35 \mathrm{E}-04$ & 0.67 & 19.26 & 38.10 \\
\hline & 29208 & $1.85 \mathrm{E}-06$ & $2.47 \mathrm{E}-05$ & $1.37 \mathrm{E}-04$ & 0.67 & 17.87 & 38.53 \\
\hline & 26931 & $1.85 \mathrm{E}-06$ & $2.38 \mathrm{E}-05$ & $1.36 \mathrm{E}-04$ & 0.67 & 17.27 & 38.37 \\
\hline & 23408 & $1.85 \mathrm{E}-06$ & $2.31 \mathrm{E}-05$ & $1.36 \mathrm{E}-04$ & 0.67 & 16.72 & 38.23 \\
\hline & 23410 & $1.85 \mathrm{E}-06$ & $2.24 \mathrm{E}-05$ & $1.35 \mathrm{E}-04$ & 0.67 & 16.20 & 38.10 \\
\hline & 20967 & $1.86 \mathrm{E}-06$ & $2.10 \mathrm{E}-05$ & $1.34 \mathrm{E}-04$ & 0.67 & 15.21 & 37.85 \\
\hline & 45223 & $1.75 \mathrm{E}-06$ & $3.84 \mathrm{E}-05$ & $1.36 \mathrm{E}-04$ & 0.63 & 27.86 & 38.43 \\
\hline & 39123 & $1.77 \mathrm{E}-06$ & $3.78 \mathrm{E}-05$ & $1.35 \mathrm{E}-04$ & 0.64 & 27.41 & 38.10 \\
\hline & 28266 & $1.80 \mathrm{E}-06$ & $3.57 \mathrm{E}-05$ & $1.37 \mathrm{E}-04$ & 0.65 & 25.89 & 38.53 \\
\hline & 26815 & $1.80 \mathrm{E}-06$ & $3.50 \mathrm{E}-05$ & $1.36 \mathrm{E}-04$ & 0.65 & 25.35 & 38.37 \\
\hline & 22744 & $1.80 \mathrm{E}-06$ & $3.42 \mathrm{E}-05$ & $1.36 \mathrm{E}-04$ & 0.65 & 24.79 & 38.23 \\
\hline & 22746 & $1.81 \mathrm{E}-06$ & $3.34 \mathrm{E}-05$ & $1.35 \mathrm{E}-04$ & 0.65 & 24.23 & 38.10 \\
\hline & 20853 & $1.81 \mathrm{E}-06$ & $3.18 \mathrm{E}-05$ & $1.34 \mathrm{E}-04$ & 0.65 & 23.09 & 37.85 \\
\hline \multicolumn{2}{|c|}{ MAX. } & $1.86 \mathrm{E}-06$ & $3.84 \mathrm{E}-05$ & $1.37 \mathrm{E}-04$ & 0.67 & 27.86 & 38.53 \\
\hline \multirow{4}{*}{ 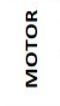 } & 64173 & $1.83 \mathrm{E}-06$ & $2.43 \mathrm{E}-05$ & $1.46 \mathrm{E}-04$ & 0.66 & 17.61 & 41.23 \\
\hline & 56567 & $1.84 \mathrm{E}-06$ & $2.72 \mathrm{E}-05$ & $1.44 \mathrm{E}-04$ & 0.67 & 19.72 & 40.64 \\
\hline & 64191 & $1.76 \mathrm{E}-06$ & $3.27 \mathrm{E}-05$ & $1.46 \mathrm{E}-04$ & 0.64 & 23.71 & 41.23 \\
\hline & 56487 & $1.76 \mathrm{E}-06$ & 3.59E-05 & 1.44E-04 & 0.64 & 26.04 & 40.64 \\
\hline & $1.84 \mathrm{E}-06$ & $3.59 \mathrm{E}-05$ & $1.46 E-04$ & 0.67 & 26.04 & 41.23 \\
\hline
\end{tabular}

\subsection{Ansys -Amplitude vs Frequency plots}

The following plots show the variance of amplitude (Ux, UY \& UZ) with frequency range of 0 to $50 \mathrm{~Hz}$ for few nodes at base locations for upper "E" value of the concrete. $X \& Z$ are TRANSVERSE and $Y$ is VERTICAL directions of ID Fan foundation. Similar plots can be generated for lower "E" values of concrete.

A) Amplitude vs frequency plots for upper E value:

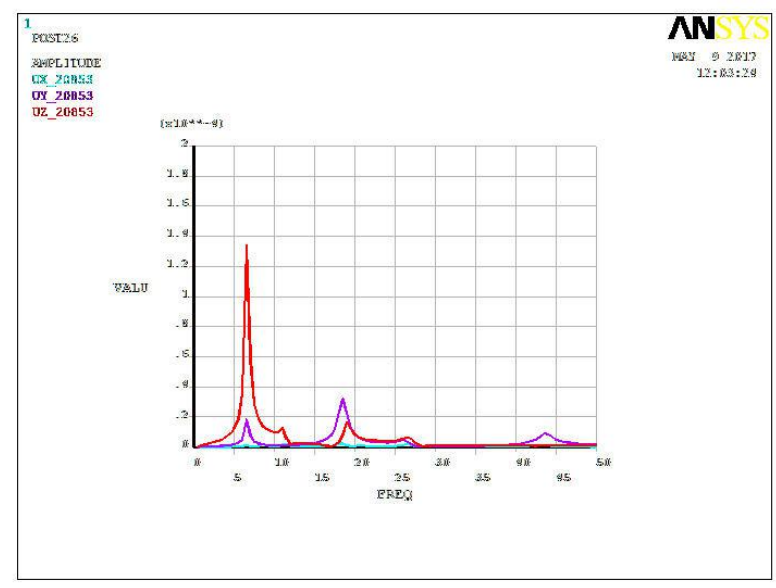

Figure 23. Vibration Amplitude Plot- Node: 20853_Ux, Uy and Uz 


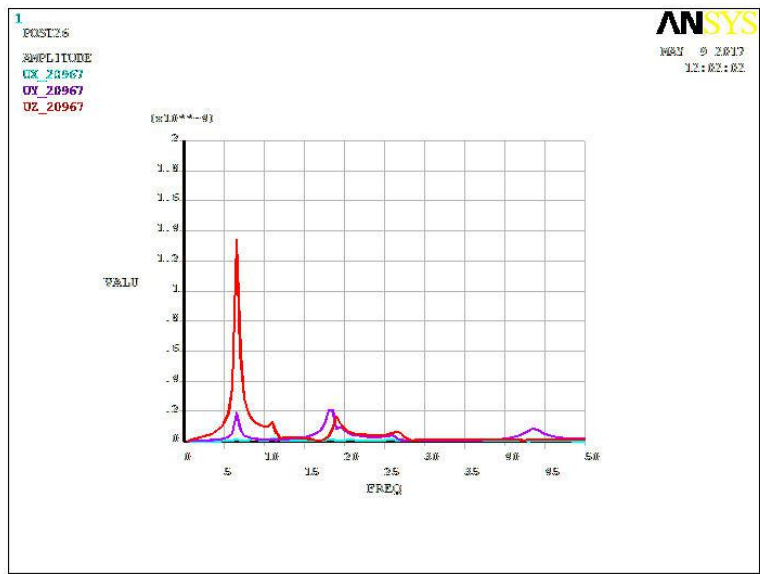

Figure 24. Vibration Amplitude Plot - Node: 20967_Ux, Uy and Uz

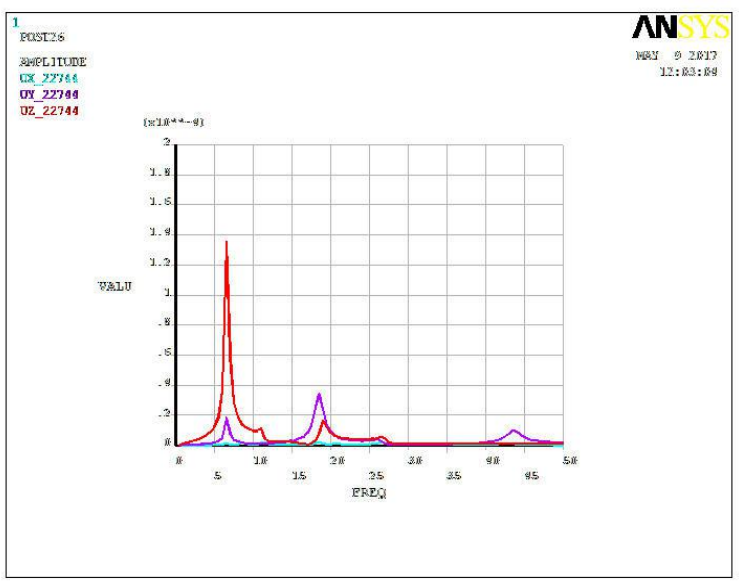

Figure 25. Vibration Amplitude Plot - Node: 22744 Ux, Uy and Uz

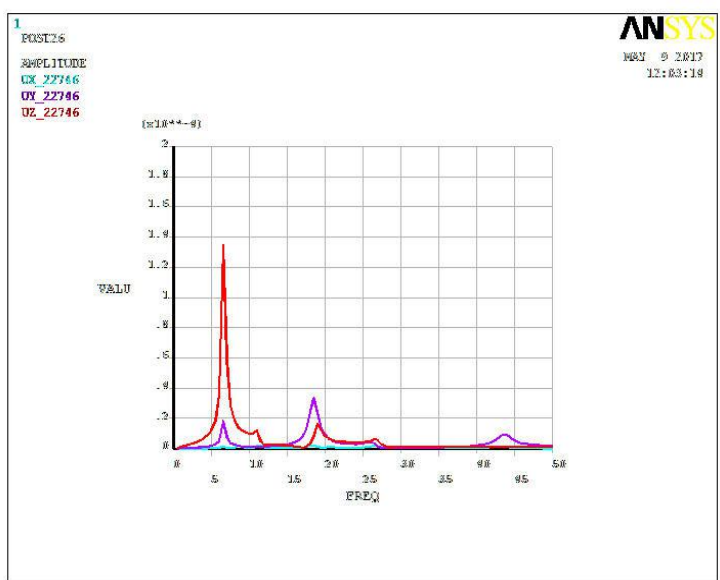

Figure 26. Vibration Amplitude Plot - Node: 22746_Ux, Uy and Uz

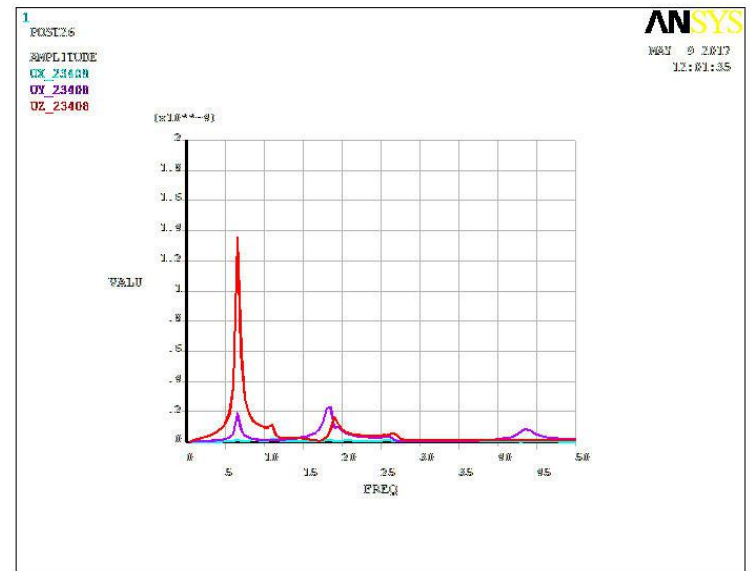

Figure 27. Vibration Amplitude Plot - Node: 23408_Ux, Uy and $\mathrm{Uz}$

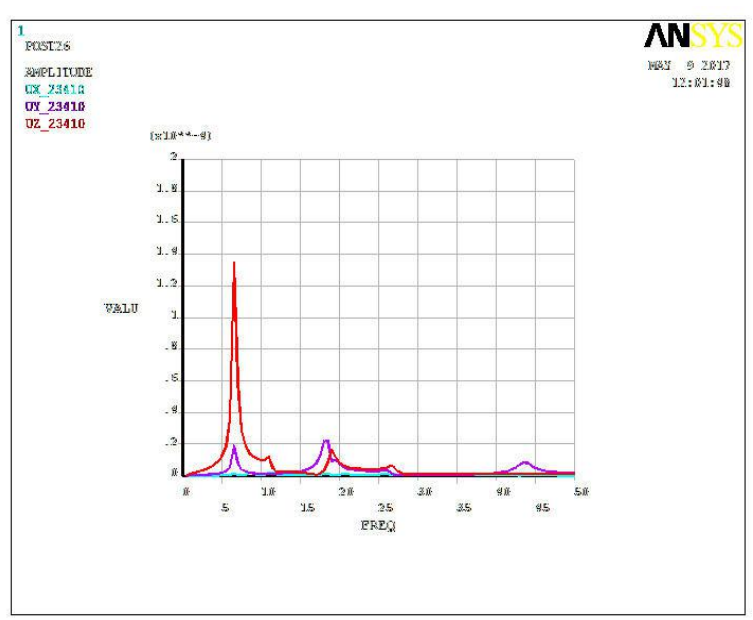

Figure 28. Vibration Amplitude Plot - Node: 23410_Ux, Uy and Uz

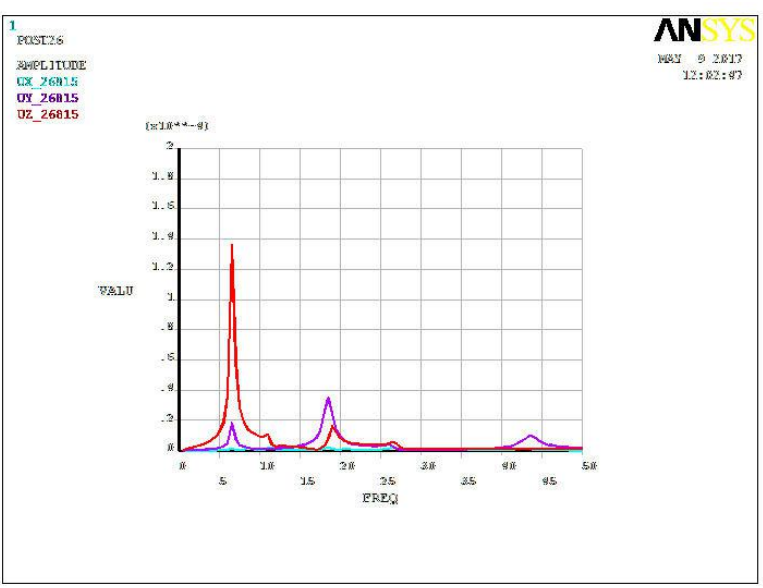

Figure 29. Vibration Amplitude Plot - Node: 26815_Ux, Uy and Uz 


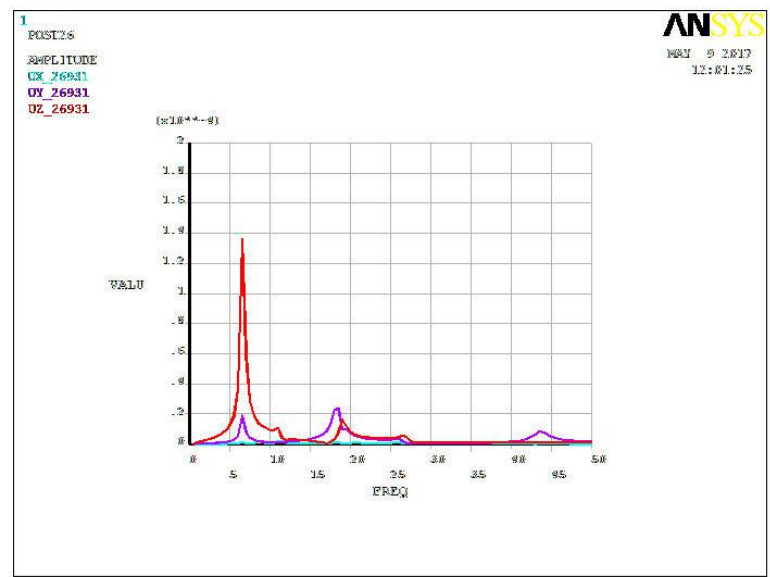

Figure 30. Vibration Amplitude Plot - Node: 26931_Ux, Uy and Uz

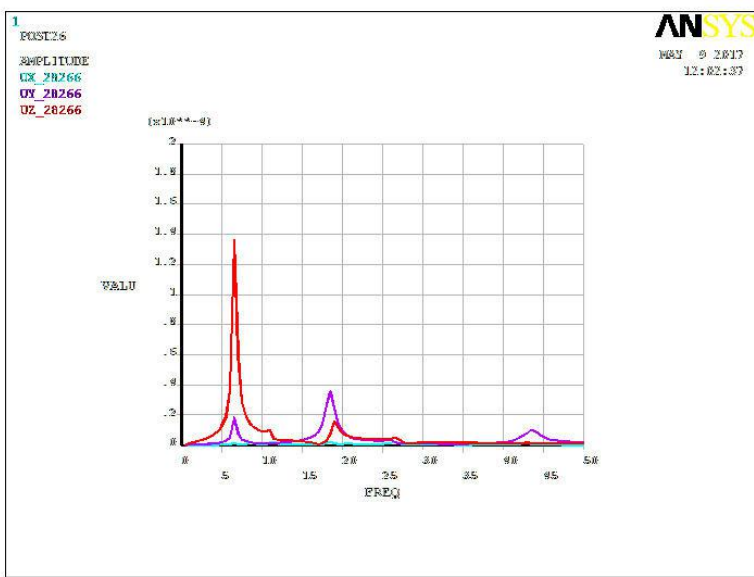

Figure 31. Vibration Amplitude Plot - Node: 28266_Ux, Uy and Uz

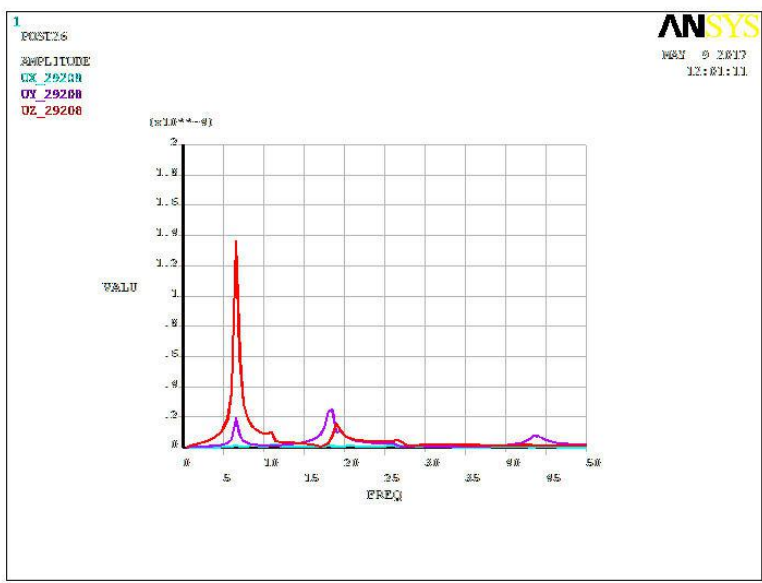

Figure 32. Vibration Amplitude Plot - Node: 29208 Ux, Uy and Uz

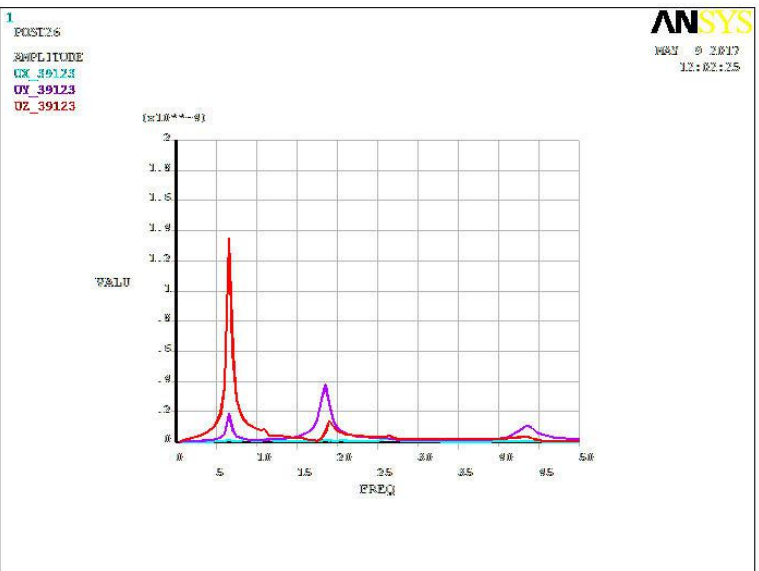

Figure 33. Vibration Amplitude Plot - Node: 39123 Ux, Uy and Uz

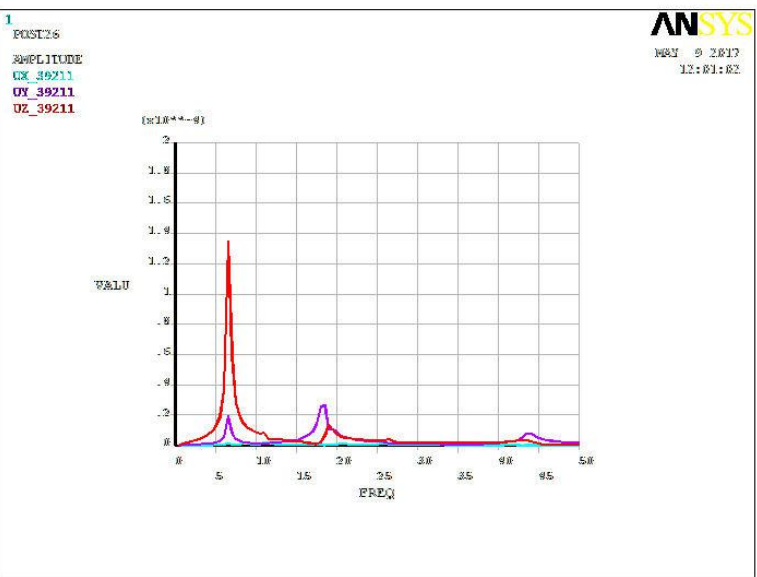

Figure 34. Vibration Amplitude Plot - Node: 39211_Ux, Uy and Uz

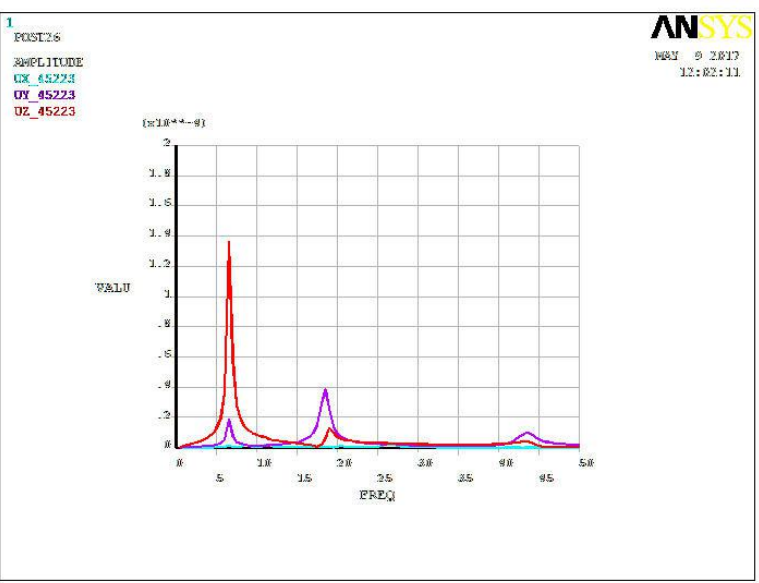

Figure 35. Vibration Amplitude Plot - Node: 45223_Ux, Uy and Uz 


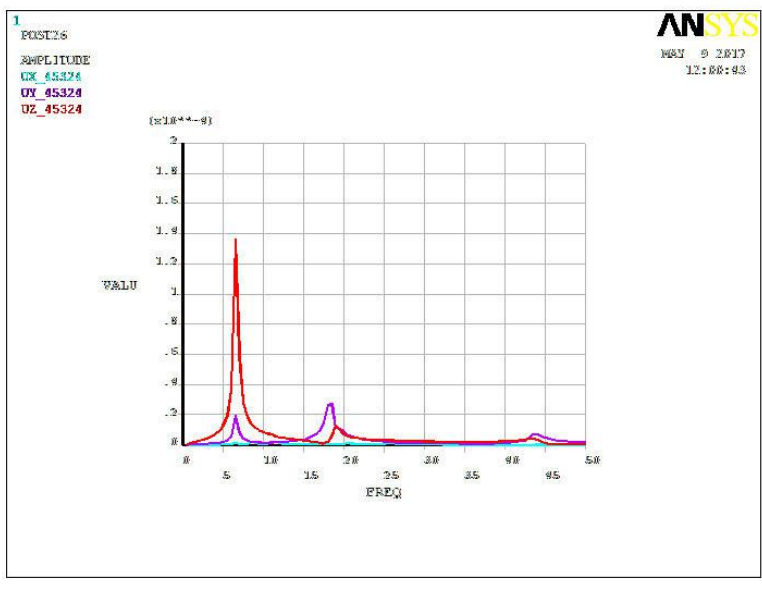

Figure 36. Vibration Amplitude Plot - Node: 45324_Ux, Uy and Uz

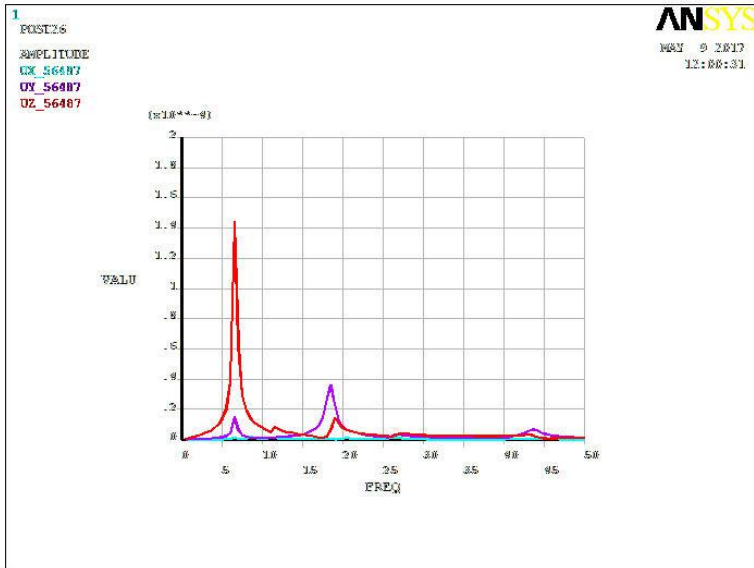

Figure 37. Vibration Amplitude Plot - Node: 56487_Ux, Uy and Uz

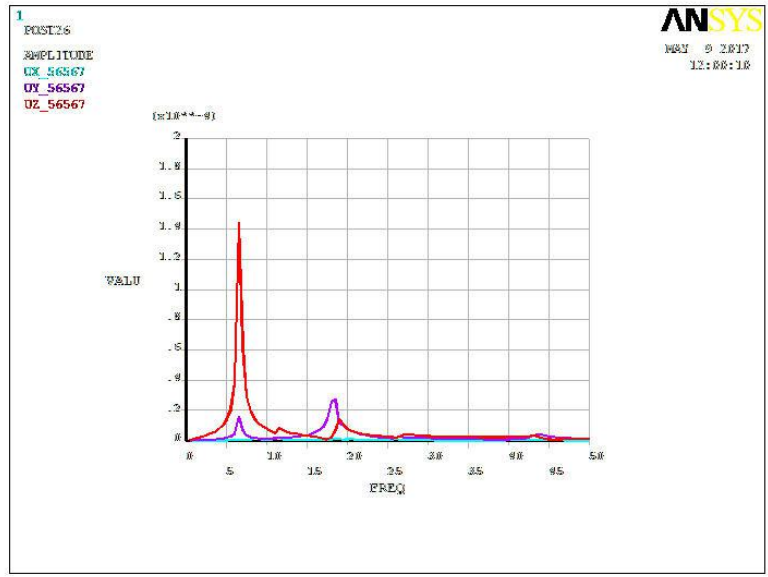

Figure 38. Vibration Amplitude Plot - Node: 56567 Ux, Uy and Uz

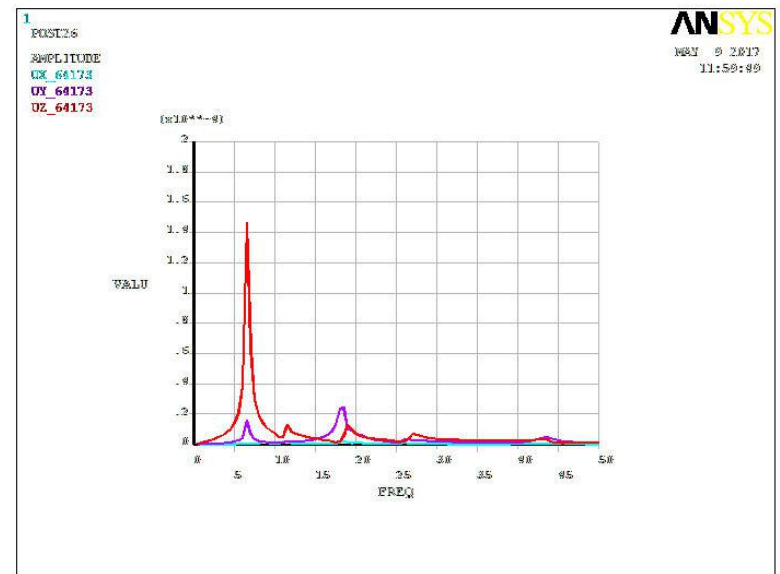

Figure 39. Vibration Amplitude Plot - Node: 64173 Ux, Uy and Uz

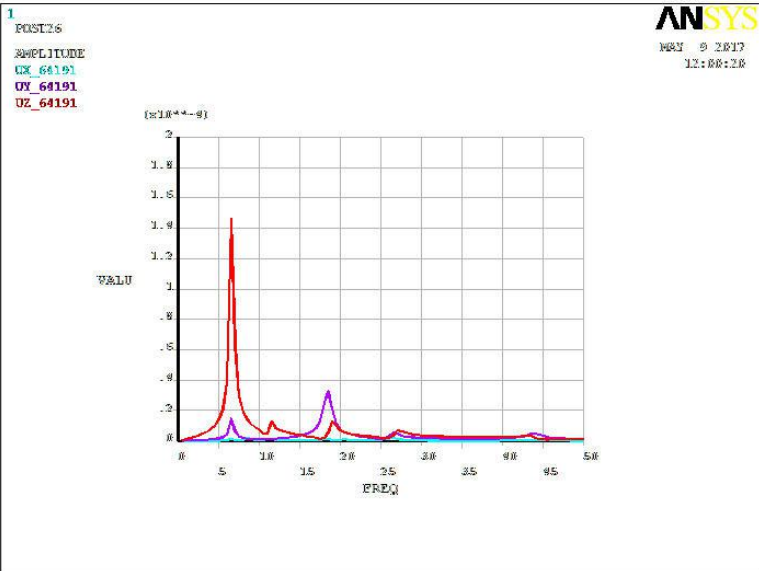

Figure 40. Vibration Amplitude Plot - Node: 64191 Ux, Uy and Uz

\section{CONCLUSIONS}

The paper highlights various issues related to the mathematical modeling of machine, foundation and soil. FEM analysis of Fan foundation described in this paper will result in safe $\&$ optimum design under static and dynamic loading conditions. Modal analysis is carried out in ANSYS software to elicit the natural frequencies of machine-foundation system for all significant modes of vibration. The absolute maximum amplitudes are obtained by performing steady state harmonic analysis of PA Fan foundation in ANSYS.

The operating frequency of the Fan and motor is 24.83 $\mathrm{Hz}$. The natural frequencies are requested for first 50 modes of vibration. But only 10 modes are present in the foundation system below $50 \mathrm{~Hz}(2.01$ times the machine operating frequency). In Upper "E" value \& Lower "E" Value the natural frequencies are away from the frequency range of the operating frequency of the Fan and motor is $24.83 \mathrm{~Hz}$. The natural frequencies 
are away from the frequency range, Fan and Motor $: 19.88 \mathrm{~Hz}(0.8 * 24.83)$ To $29.80 \mathrm{~Hz}(1.2 * 24.83)$. Hence there by, resonance condition is avoided.

Frequency vs Amplitude plots for the variance of amplitude (Ux, Uy \& Uz) with frequency $(0 \sim 50 \mathrm{~Hz})$ for different nodes at equipment base locations are generated. These plots helped in understanding the foundation behaviour during equipment start-up \& shut down transient conditions apart from the operating condition. The peak values shown in the plots represents the transient stage resonant condition's which are many times more than the amplitude values at operating condition's. This helps engineers to consider any precautionary measures during transient conditions. All the amplitudes at base points of motor and Fan on the top of the foundation under operating frequencies $(24.83 \mathrm{~Hz})$ are well with in the allowable value of $200 \mu \mathrm{m}$ and the Rating of the foundation is "Good" as per VDI 2056

This paper will also help the structural engineer practitioners in design of PA Fan foundation's by applying Finite Element Methods using ANSYS software.

\section{REFERENCES}

[1] N. Leso, J. Puttonen, E. Porkka, The effect of foundation on fan vibration response, J. Struct. Mech. 44 (1) (2011) 120.

[2] D.R. Smith, H.R. Simmons, Unique fan vibration problems: their causes and solutions, in: Proceedings of the 9th Turbomachinery Symposium Gas Turbine Laboratories, 1980.

[3] D. Smith, J. Wachel, Controlling fan vibration-case histories, in: EPRI Symposium on Power Plant Fans: The State of the Art,1981.

[4] R. Galindo, M. Illueca, R. Jimenez, Permanent deformation estimates of dynamic equipment foundations: application to a gas turbine in granular soils, Soil Dyn. Earthq. Eng. 63 (2014) $8-18$.

[5] M. Dias Jr., K.L. Cavalca, Experimental analysis of the dynamic behavior of a turbomachine foundation structure, rn 8 (1) (1999).

[6] M. Zaaijer, Foundation modelling to assess dynamic behaviour of offshore wind turbines, Appl. Ocean Res. 28 (1) (2006) 45-57.

[7] Design of structures and foundation for vibrating machines by Surech Arya, Michel O' Neil, Gorge Pincus 